\title{
Development
}

\section{Forebrain Neural Precursor Cells Are Differentially Vulnerable to Zika Virus Infection}

\author{
DSamantha M. Shelton, ${ }^{1,2}$ Alexandra R. Soucy, ${ }^{3}$ Ronni Kurzion, ${ }^{1}$ Ella Zeldich, ${ }^{1}$ John H. Connor, ${ }^{3}$ and \\ (DTarik F. Haydar ${ }^{1,4}$
}

https://doi.org/10.1523/ENEURO.0108-21.2021

${ }^{1}$ Department of Anatomy and Neurobiology, Boston University School of Medicine, Boston, MA 02118, ${ }^{2}$ Graduate Program in Neuroscience, Boston University School of Medicine, Boston, MA 02118, ${ }^{3}$ Department of Microbiology and National Emerging Infectious Diseases Laboratories, Boston University Medical Campus, Boston, MA 02118, and ${ }^{4}$ Center for Neuroscience Research, Children's National Hospital, Washington, DC 20010

\begin{abstract}
Prenatal exposure to Zika virus (ZIKV) can result in microencephaly and congenital Zika syndrome, although some brain cells and structures are spared by the virus for unknown reasons. Here, a novel murine model of fetal ZIKV infection incorporating intraventricular infection and cell type-specific in utero electroporation (IUE) was used to identify the time course of ZIKV infection and to determine the identity of cells that are initially infected or spared during neocortical neurogenesis. In vivo time course studies revealed the presence of ZIKV in apical radial glial cells (aRGCs) at early time points following virus exposure, while basal intermediate progenitor cells (bIPCs) became maximally $\left(\mathrm{ZIKV}^{+}\right)$after $3 \mathrm{~d}$ of virus exposure. ZIKV-infected fetal brains exhibited microencephaly as early as $1 \mathrm{~d}$ following infection, regardless of developmental age. This change in brain size was caused in part by apoptosis and reduced proliferation that persisted until birth. While $60 \%$ of aRGC basal fibers were perturbed during infection, $40 \%$ retained normal morphology, indicating that aRGCs are not uniformly vulnerable to ZIKV infection. To investigate this heterogeneous vulnerability, we performed genetic fate mapping using cell type-specific probes derived from a mouse embryonic day (E) 15.5 neocortical wall singlecell RNA sequencing (scRNAseq) dataset. The results indicate that one class of aRGCs preferentially express the putative ZIKV entry receptor $A X L$ and that these cells are more vulnerable to ZIKV infection than other aRGC subtypes with low $A X L$ expression. Together, these data uncover crucial temporal and cellular details of ZIKV fetal brain infection for prevention strategies and for management of congenital Zika syndrome.
\end{abstract}

Key words: corticogenesis; development; heterogeneity; microcephaly; radial glia; Zika

\section{Significance Statement}

The transcriptional signatures of neural precursor cells (NPCs) were used for the first time to test Zika virus (ZIKV) susceptibility in a direct fetal brain infection model. This novel methodology allowed for elucidation of time point-specific differences in NPC susceptibility that have been debated in the field. Additionally, elucidation of cell morphologic features using in utero electroporation (IUE) revealed substantial but incomplete interruption of basal fibers, a finding that implies interference with neuronal migration. The model presented here, allows for assessment of prenatal development after exposure to a variety of viruses. The improved specificity of apical radial glial cell (aRGC) labeling afforded by the cell-specific labeling tools uncover functional differences between aRGC types that will have important implications for children exposed to ZIKV as well as for understanding corticogenesis.

Received March 17, 2021; accepted July 8, 2021; First published July 16, 2021.

The authors declare no competing financial interests.
Author contributions: S.M.S., J.H.C., and T.F.H. designed research; S.M.S., A.R.S., R.K., E.Z., and T.F.H. performed research; S.M.S. and A.R.S. analyzed data; S.M.S. and T.F.H. wrote the paper. 


\section{Introduction}

The 2015 Brazilian Zika virus (ZIKV) outbreak has been associated with an estimated 20 -fold increase in cases of microcephaly in newborns (Center for Disease Control, 2017). Despite the strong association between prenatal ZIKV exposure and the devastating effects of microcephaly, there are no available treatments or approved vaccines for ZIKV despite the rapid response by the biomedical community (CDC, 2019). Greater understanding of the mechanisms underlying ZIKV-induced microcephaly is needed to aid in the development of therapeutic strategies. In particular, the infection route and cellular mechanisms contributing to ZIKV-induced microcephaly are still unclear. The possibility that cells are not uniformly affected by virus exposure may lead to new therapies or prevention strategies.

Various approaches have been used to understand the mechanisms behind ZIKV-induced microencephaly, including cell culture, organoids, slice culture, and animal models (Haubensak et al., 2004; Bhattacharyya et al., 2013; Gadea et al., 2016; Lazear et al., 2016; Miner and Diamond, 2016; Nowakowski et al., 2016; Onorati et al., 2016; Shao et al., 2016; Tang et al., 2016; Dudley et al., 2017; Meertens et al., 2017; Rosenfeld et al., 2017; Vanwalscappel et al., 2018; Politi et al., 2020). Major obstacles for model development have included the differences in immune responses between humans and rodents. While ZIKV exhibits tropism in human maternal and fetal epithelium during gestation, the maternal immune system and placental barrier in mice prevents fetal infection (Lazear et al., 2016). Interferon knock-out mice allow maternal-fetal transmission of ZIKV, thus serving as a potential system of study (Lazear et al., 2016). A challenge of this model is that the loss of interferon likely alters the natural progression of infection, cell death, and proliferation in the central nervous system, impairing the translatability of findings from these models (Bhattacharyya et al., 2013; Meertens et al., 2017; Vanwalscappel et al., 2018). Studies using 1D, 2D, and 3D stem cell cultures have demonstrated key aspects of ZIKV infection, including increased cell death and decreased proliferation, but these methods do not contain the full repertoire of cell types or developmental processes necessary for proper brain development (Gadea et al., 2016; Miner and Diamond, 2016; Tang et al., 2016; Meertens et al., 2017). Similarly, while assessments using in utero electroporation (IUE) have identified morphologic changes to precursors and neurons in response to ZIKV, they have not reached cell type-specific resolution (Rosenfeld et al., 2017). Several other studies have been performed with inactivated ZIKV or

This work was supported by the National Institutes of Health Grant 1R21NS101151-01.

Correspondence should be addressed to Tarik F. Haydar at thaydar@ childrensnational.org.

https://doi.org/10.1523/ENEURO.0108-21.2021

Copyright @ 2021 Shelton et al.

This is an open-access article distributed under the terms of the Creative Commons Attribution 4.0 International license, which permits unrestricted use, distribution and reproduction in any medium provided that the original work is properly attributed. with one of the first available strains of the virus, MR766 (Gadea et al., 2016; Tang et al., 2016). Conclusions from these studies exhibited lack of viral transmission and attenuating mutations within research stocks of MR-766. To overcome these obstacles, we sought to improve on the IUE infection model that circumvents the dam's immune defenses, permitting study of infection from a contemporary strain of ZIKV over the period of cortical development in utero.

We have combined this direct brain infection model with cell type-specific methods to determine which groups of neural precursor cells (NPCs) are most susceptible to prenatal ZIKV exposure. In the mouse neocortex, all lineages of neurons are born between embryonic day (E)11 and E17 from a diverse population of precursor cell types. These neurons are generated either directly or indirectly from a secondary progenitor. This period of cortical expansion is essential to brain development, and the ratio of direct and indirect neurogenesis has a large impact on the eventual size and neuronal complexity of the neocortex (Rakic, 1987, 1988, 1995). Thus, viral effects on individual NPC populations could result in smaller populations of their specific neuronal progeny, resulting in microencephaly and dysgenesis of particular cell populations in the cortical architecture. Previous studies have shown that apical radial glial cells (aRGCs) are preferentially targeted by ZIKV while others have suggested that basal intermediate progenitor cells (bIPCs) are the target of infection (Onorati et al., 2016; Lin et al., 2017). Emerging data on differences between progenitor cell groups might be crucial in elucidating nuanced mechanisms of ZIKV infection and consequent changes in neuronal output. NPCs can be categorized into sub-classes by transcriptional profile, morphology, and location within the ventricular zone (VZ) and sub-VZ (SVZ; Fig. 1A; Rakic, 1972; Hartfuss et al., 2001; Tamamaki et al., 2001; Holsapple et al., 2003; Haubensak et al., 2004; Miyata et al., 2004; Noctor et al., 2004; Englund et al., 2005; Tyler et al., 2015; Tasic et al., 2018; Hodge et al., 2019; Heavner et al., 2020; Li et al., 2020). Recent advancements in single-cell RNA sequencing (scRNAseq) provide the opportunity to study the heterogeneity of NPCs and their daughter cells at new levels of resolution. We sought to make use of these transcriptomic distinctions between NPCs to determine the cell type-specific infection profile of ZIKV over time in vivo.

We therefore developed a method to directly infect the telencephalon of a non-immunocompromised mouse model with a contemporary strain and physiological titer of active ZIKV with low passage number. To study subtypes of NPCs in the infected developing brain, we performed IUE during ZIKV exposure (Fig. 1B). This technique enabled us to study the infection process in apical precursors (aRGCs, apical IPCs) and basal precursors (bIPCs and basal RGCs) over time in vivo (Tyler et al., 2015). These findings demonstrate the effective use of a novel in vivo method for studying the effects of ZIKV infection on the developing brain, and they 
A

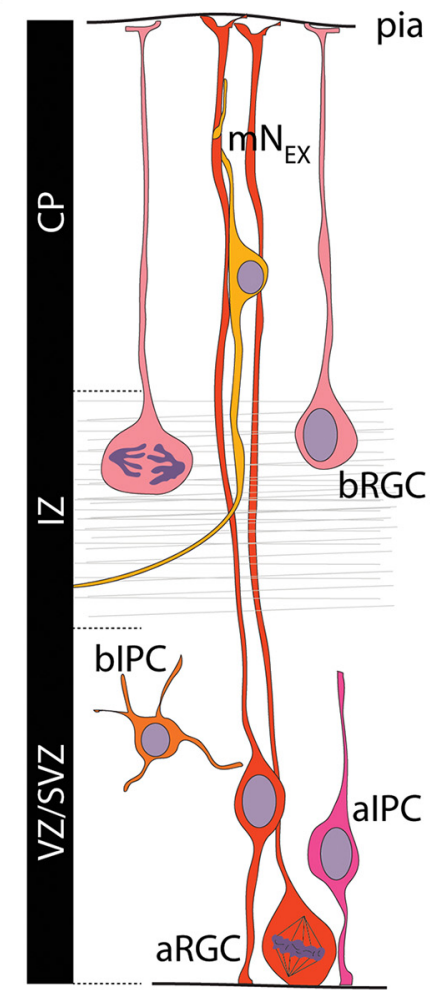

B

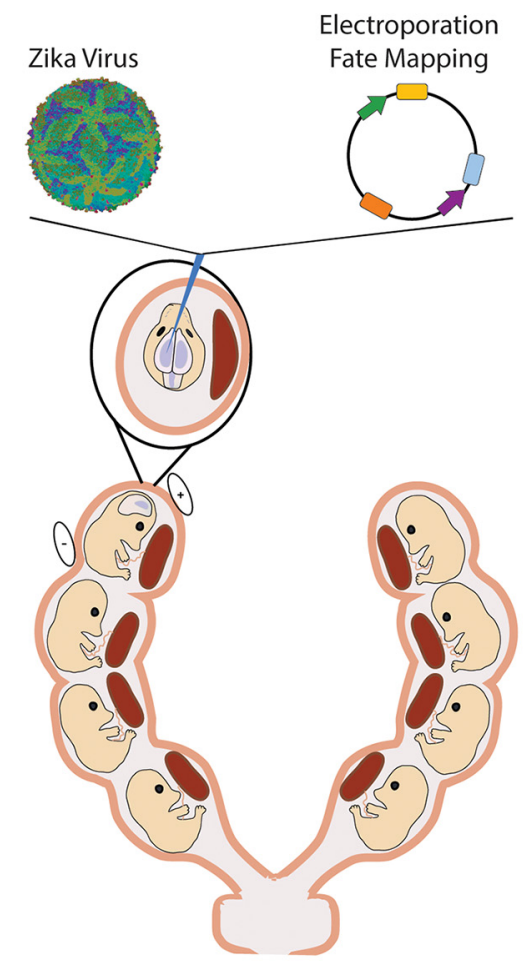

C

ventricle

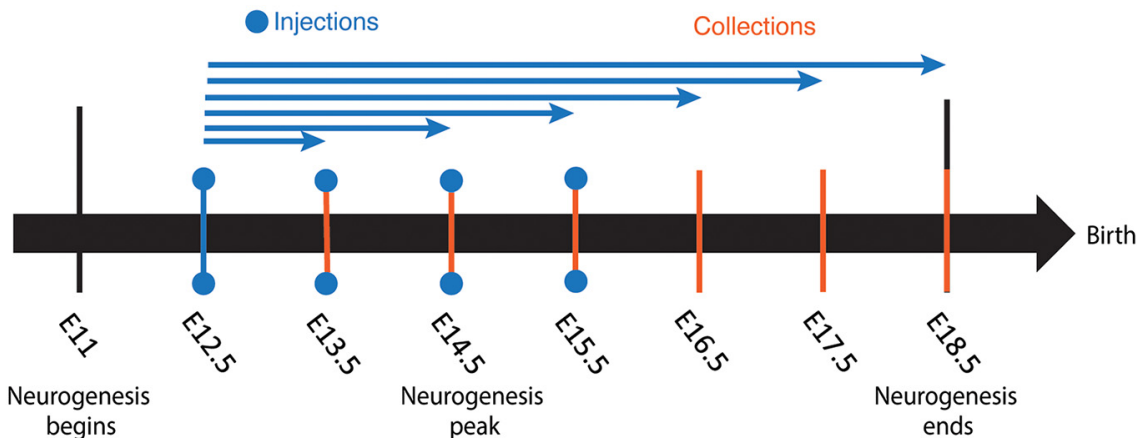

Figure 1. Intraventricular ZIKV model and genetic fate mapping approach. $\boldsymbol{A}$, Each NPC of the cerebral cortex has a unique transcriptional profile, morphology, and location of mitosis. $\boldsymbol{B}$, A total of 10,000 FFU ZIKV or saline, and cell type-specific plasmid DNA are injected into the lateral ventricle. C, IUE and ZIKV infection experiments include collection on E13.5-E18.5 encompassing early, $\mathrm{mid}$, and late neurogenesis. The blue arrows denote the temporal infection-collection plan for the E12.5 experiments for example.

provide new insight into corticogenesis in children affected by virus-induced microencephaly.

\section{Materials and Methods}

All materials and supplies are listed in Table 2 and all statistical results are presented in Table 3.

\section{ZIKV production}

Vero E6 cells were maintained and passaged in cMEM [MEM (Invitrogen) containing 10\% fetal bovine serum (Sigma Life Science)]. The ZIKV PRVABC59 (2015 Puerto Rico strain, GenBank KZ087101.2) was obtained from BEI resources. Master stocks were prepared from supernatants of Vero E6 cells infected at low $\mathrm{MOI}$ as published in Rossignol et al. (2017).

\section{Animals and IUE}

IUE was performed on timed pregnant CD-1 dams purchased from Charles River Laboratories. Dams were separated into individual cages on arrival to prevent overcrowding. Animals were allowed to rest for $72 \mathrm{~h}$ in standard cages with huts and a ZT12. Dams were anesthetized via intraperitoneal injection of a ketamine/xylazine mixture $(10 \mathrm{mg} / \mathrm{kg})$, and the uterine horns were exposed by midline laparotomy. One to two microliters of plasmid DNA mixed with $0.1 \%$ fast green dye (Sigma-Aldrich) was injected intracerebrally, through the uterine wall and 
amniotic sac, via a pulled glass micropipette. Cre and reporter plasmid DNA was mixed at a 1:1 ratio by copy number, and the final concentration of each plasmid was between 2 and $3 \mu \mathrm{g} / \mu \mathrm{l}$. The anode of a Tweezertrode (Harvard Apparatus) was placed over the dorsal telencephalon above the uterine muscle, and four $35-\mathrm{V}$ pulses (50-ms duration separated by a 950-ms interval) were applied with a BTX ECM830 pulse generator (Harvard Apparatus). Following electroporation, the uterine horns were returned to the abdomen and the cavity was filled with a warm $0.9 \%$ sterile saline solution. The incisions were closed with silk or absorbable sutures depending on the duration of the experiment. Dams were then placed in a clean cage and monitored closely during recovery. After either 24 or $48 \mathrm{~h}$ mice were killed with $\mathrm{CO}_{2}$ asphyxiation and cervical dislocation. Male and female pups were removed from the uterus and decapitated before drop fixation. All animal procedures were performed in accordance with the Boston University animal care committee's regulations.

To model infection of a contemporary ZIKV in fetal brain, we chose to directly inject the PRVAB59 strain into the lateral ventricle of prenatal mice in utero. The concentration was determined after initial tests of infectability at concentrations ranging from 1000 to $12,000 \mathrm{FFU}$. We used the lowest concentration able to cause reliable infection to better model the expected viral load that would be transmitted by infected mothers to human fetuses. The average bolus from the Aedes aegypti mosquito is $10^{4}-10^{8} \mathrm{FFU}$. Some of that bolus is cleared from the maternal blood stream before infecting a fetus, making the expected titer around $10^{1-3}$ FFU (Dudley et al., 2017). $10^{3}$ FFU was sufficient to cause infection in mice without causing large scale cell death. Antibodies to ZIKV envelope protein resulted in false positives in western blot, therefore antibody against non-structural protein $2 \mathrm{~b}$ (NS2B) was used for all experiments. To infect embryos at the time of IUE, $10^{3} \mathrm{FFU}$ was added to the plasmid DNA and fast green dye cocktail in place of saline. Embryos in one uterine horn were injected with plasmid DNA cocktail containing virus and embryos in the contralateral uterine horn were injected with plasmid DNA cocktail containing saline to provide an age-matched vehicle control. Immunohistochemistry $(\mathrm{IHC})$ against ZIKV and RT-qPCR were conducted to ensure that ZIKV did not infect the control embryos. These procedures were reviewed and approved by the Institutional Animal Care and Use Committee at the Boston University.

\section{Cloning}

The cloning into the recipient Cre recombinase vector was done using In-Fusion technology and the backbone vector carrying Cre was amplified using primers forward, CTCGAGGGGCAGAGCC; reverse, GGTACCCAATTCGC CCTATAGTGAG (Tyler and Haydar, 2013).

A 2.9-kb fragment of DNA upstream of the Prc1 translation initiation (Li et al., 2004) site was amplified by PCR from mouse genomic DNA with CloneAmp HiFi PCR Premix (TakaraBio) using the following primers with the added 15-bp overlap at their ends: forward, TACCGTT
CTCCGTCCCGCTCGAGGGGCAGAGCCG; reverse, GC TCTGCCCCTCGAGCGGGACGGAGAACGG; forward, GG CGAATTGGGTACCCTTGGCTTGCTAGGGTGTGA; reverse, CCCTAGCAAGCCAAGCGCTATC). The 3.2-kb Robo4 promoter (Okada et al., 2015) was amplified with the same overlapping ends using forward, GGCGAATTGGGTAC CCATGCATTTGGAGTTTCCATGTCCT and reverse, GC TCTGCCCCTCGAGGGCTGCTCTCGGCTCC. The 424bp MFAP2 promoter (add 16321658) was amplified the same way using primers forward, GGCGAATTGGGT ACCACTCGATCTCCCTTAATCTGCCT; reverse, GGC GAATTGGGTACCACTCGATCTCCCTTAATCTGCCT.

Amplified DNA for the inserts and the vector was purified with a NucleoSpin PCR Clean-UP kit (TakaraBio) and 25-50 ng of DNA were fused together using In-Fusion HD enzyme premix (TakaraBio). The fused product was transformed into chemically competent Stellar competent cells (TakaraBio) for selection by growth on LB Amp agar plates. Cloning was confirmed by DNA sequencing.

\section{RT-qPCR}

RNA was extracted from flash frozen tissue with a QIAGEN RNeasy kit per manufacturer recommendations. Quantitative RT-qPCR was conducted with a Roche onestep SYBR ZsGreen kit. Primers used were ZIKV forward, AARTACACATACCARAACAAAGTGGT and ZIKV reverse, TCCRCTCCCYCTYTGGTCTTG.

\section{Lipophilic staining}

Dil $(1 \mathrm{mg} / \mathrm{ml})$ was dissolved in $100 \%$ ethanol and allowed to air dry on a Petri dish until crystals formed $(\sim 1 \mathrm{~h})$. Meninges were removed from brains that were fixed in $4 \%$ paraformaldehyde (PFA) overnight. The dorsal surface of the brain was rolled over the bed of Dil crystals to create a uniform coating. The labeled brains were stored in $0.02 \%$ sodium azide in PBS, light protected at room temperature for two weeks. Brains were mounted in $4 \%$ agarose and cut into $80-\mu \mathrm{m}$ sections with a Leica VT1000S vibrating microtome; $75-\mu \mathrm{m}$ Z-stack images were taken using an upright Zeiss LSM 710 microscope. Cells were manually scored and counted using the LSM image browser software.

\section{IHC}

Embryos were collected at 1-6 d postinfection (DPI), and the heads were fixed overnight in 4\% PFA, cryoprotected in $30 \%$ sucrose for $24-48 \mathrm{~h}$, and frozen in OCT compound in tissue molds with an ethanol/dry ice bath. Frozen tissue was cut into $16-\mu \mathrm{m}$ sections using a HM 550 Cryostar cryostat and mounted and dried onto superfrost slides.

Before staining, frozen sections were air dried for $1 \mathrm{~h}$ and washed three times in PBS for 5 min each. Sections were then blocked in diluent ( $5 \%$ goat serum, $0.3 \%$ Triton $\mathrm{X}-100,1 \times \mathrm{PBS}$ ) for $1 \mathrm{~h}$ at room temperature.

Incubation with primary antibodies, mouse anti-Ki67 (1:250, Thermofisher), rabbit anti-phospho-histone H3 (PH3; 1:300, Millipore), rabbit-Tbr2 (1:500, Millipore), goat anti-Sox2 (1:250, Santa Cruz), rabbit-NS2B (1:250, 
Genetex), and rabbit anti-cleaved caspase 3 (CC3; 1:250, Cell Signaling) were performed overnight at room temperature. Following three 5-min washes in PBS, sections were incubated for $2 \mathrm{~h}$ at room temperature in diluent containing the appropriate Alexa Fluor-conjugated secondary antibodies (1:500 for all). Sections were washed an additional three times for $5 \mathrm{~min}$ and stained with Hoechst at 1:1000 in PBS for $10 \mathrm{~min}$. Slides were washed with $1 \times$ PBS three times for 5 min and coverslipped with fluoromount-G. The $20 \times Z$-stack images $(20 \mu \mathrm{m})$ were acquired using an upright Zeiss LSM 710 microscope and positive cells from $n=4$ brains were identified and counted using LSM image browser software.

ZsGreen $^{+}$and mCherry ${ }^{+}$cells from electroporation were manually scored and counted using the LSM image browser software. Cells were classified as "ZsGreen " if they expressed ZsGreen but contained no signal for mCherry. Correspondingly, cells were classified "mCherry" " if they contained mCherry signal, whether or not they were also ZsGreen $^{+}$because of perdurance of the fluorescent protein, remaining copies of unrecombined reporter plasmid, or because of the presence of a subpopulation of precursors transitioning from an apical to a basal fate.

\section{Fluorescent in situ hybridization}

Fluorescent in situ hybridization was performed using the RNAscope (ACDbio) protocol for fixed frozen sections with the modification of using 100\% methanol with 3\% hydrogen peroxide rather than using $100 \%$ peroxide to preserve the integrity of the tissue. The protocol can be found on the ACDbio webpage (https://acdbio.com/technicalsupport/user-manuals) under user manual document number 320293-USM. Cells were counted as being positive if they had two or more puncta co-localized to the nucleus labeled with Hoechst. The $20 \times Z$-stack images $(20 \mu \mathrm{m})$ were acquired using an upright Zeiss LSM 710 microscope and positive cells from $n=4$ brains were identified and counted using LSM image browser software.

\section{Analysis of differentially expressed genes from scRNAseq data}

Genes that had high expression in only one group and that were expressed in the majority of cells within a group were then assessed for location of gene expression. The online Allen Brain Atlas was used to investigate in situ hybridization of genes of interest to verify expression in the VZ of developing mouse brain (Allen Brain Developing Mouse Brain Atlas, http://developingmouse.brain-map. org). From there, a short list was generated of genes that showed a significant difference in gene expression between aRGC groups 1 and 2, that had high expression in many cells within their cluster and showed gene expression localized to the VZ of the developing murine cortex. Markers who showed expression in cell types other than aRGCs and that were expressed outside of the VZ at E15.5 were excluded. Additionally, the find markers algorithm in the Seurat package offered in $R$ was used to corroborate this list. Only the two most specific markers were used for each aRGC group.

\section{Statistics}

A two-way Student's $t$ test was run for the log fold change of each gene in aRGC groups 1 and 2 to compare differential gene expression between the two groups. Histograms of $z$ scores were generated for genes that reached significance. Genes that had high expression in only one group and that were expressed in the majority of cells within a group were then assessed for location of gene expression. These top candidate markers were secondarily validated with the Seurat Find Markers algorithm.

Cell counts were analyzed in Microsoft excel and Prism using a two-way Student's $t$ tests with a $p<0.05$ considered statistically significant when comparing control and infected samples. When comparing statistical differences among the means of two or more groups, a one-way ANOVA was performed in Prism with $p<0.05$ considered statistically significant.

\section{Results}

\section{Active ZIKV injection into the lateral ventricle of the prenatal mouse brain}

To model infection of a contemporary ZIKV strain in the fetal brain, 10,000 FFU of the PRVAB59 strain was injected into the lateral ventricle of prenatal mice in utero. This concentration was determined after initial tests of infectability within a 5000-12,000 FFU range. We used the lowest concentration necessary to cause reliable infection to better model the expected viral load that would be transmitted by infected mothers to human fetuses. The average bolus from the $A$. aegypti mosquito is $10^{1-3} \mathrm{FFU}$ (Dudley et al., 2017). We found that $10^{3} \mathrm{FFU}$ was sufficient to cause reliable infection in the fetal mouse brain. $\mathrm{IHC}$ for NS2B was used to test ZIKV viral replication for all experiments. IHC using NS2B was employed to test for the presence of replicating ZIKV in control and infected embryos at various times following infection (Fig. 2). IHC (Fig. $2 A-E$ ) and RT-qPCR (Fig. $2 F$ ) studies were conducted to determine that the virus did not pass across the cervix to cause infection in the contralateral uterine horn, which was used for age-matched saline vehicle controls. Widespread infection was low at 1 DPI and steadily increased in the following days. By 6 DPI, most virus was present in the cortical neuron layers in the VZ, indicating that ZIKV had been cleared from the cerebral spinal fluid of the lateral ventricles and was replicating in cells located away from the ventricle by this time point (Fig. 2E).

\section{Time course of ZIKV-induced microencephaly}

To determine whether this mouse model of fetal ZIKV infection recapitulates the microencephaly phenotype found in humans and animal models, measurements of control and infected littermate brains were collected over time. In saline-infected or virus-infected littermates, we calculated brain size rather than head circumference to use a more reliable and repeatable method of measuring microencephaly. Control and infected brains were 

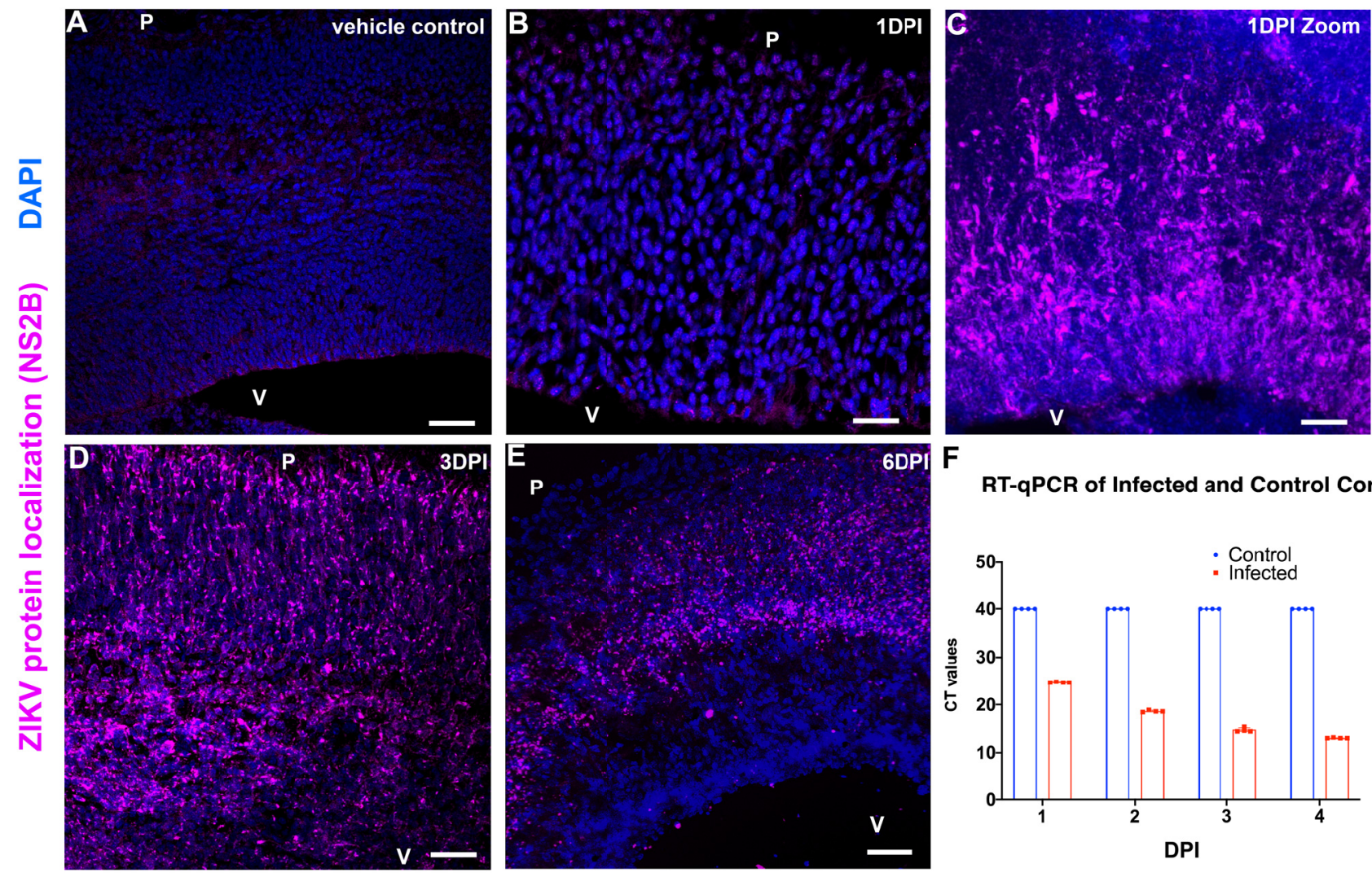

RT-qPCR of Infected and Control Cortex

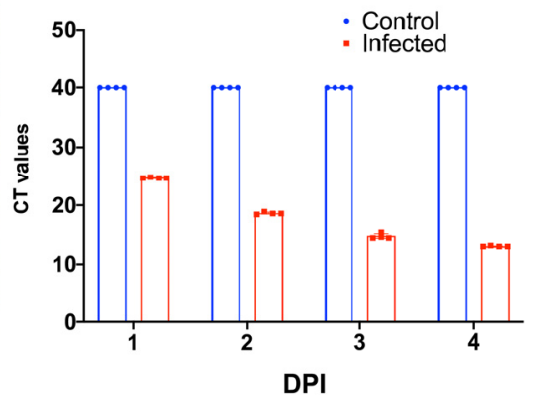

Figure 2. Fetal ZIKV infection spreads radially throughout the telencephalon. NS2B immunofluorescence in fetal brains infected intraventricularly on E12.5 with 10,000 FFU of ZIKV and collected 1, 3, and 6 DPI. A, Vehicle control neocortical wall at 3 DPI. Scale bar: $60 \mu \mathrm{m}$. B, Infected neocortical wall at $1 \mathrm{DPI}$. Scale bar: $30 \mu \mathrm{m}$. $\boldsymbol{C}$, High-magnification image of infected neocortical wall at 1 DPI. Scale bar: $10 \mu \mathrm{m}$. $\boldsymbol{D}$, Infected neocortical wall at $3 \mathrm{DPI}$. Scale bar: $60 \mu \mathrm{m}$. E, Infected neocortical wall at 6 DPI. Scale bar: $60 \mu \mathrm{m}$. $\boldsymbol{F}$, Presence of ZIKV was also measured with RT-qPCR in vehicle-injected and ZIKV-injected fetal brains at 1, 2, 3, and 4 DPI. The vehicle-injected brains had RT-qPCR cycle thresholds (CTs) at or above 40 cycles, indicating no presence of virus. The number of amplification cycles necessary to detect ZIKV decreased with each day after injection $(n=4,4)$.

measured along the medial-lateral and rostral-caudal axes to assess the differences in forebrain size (Fig. 3A). When fetuses were injected at E12.5, microencephaly $(>2$ SDs below the mean) was found at all examined time points (1-6 DPI; Fig. 3B,C). In fact, viral infection on $\mathrm{E} 12.5,13.5,14.5$, or 15.5 generated microencephaly quickly, as early as $1 \mathrm{DPI}$. These time points span neurogenesis and infection times analogous to the second trimester of human gestation. Surprisingly, the ZIKVinduced decrease in brain size was not dependent on the age of infection when testing 1 and 2 DPI infection at E12.5, E13.5, E14.5, and E15.5 (Fig. 3D-G). Additionally, cortical thickness, measured at each time point after E12.5 infection, was consistently decreased from 1DPI to birth (example images of vehicle control in Fig. $3 \mathrm{H}$, infected in Fig. 3/, and analysis in Fig. 3J). The $\sim 100-\mu \mathrm{m}$ decrease in gross brain size was in line with the extent of reduction in cortical thickness, indicating that the decrease in brain size is primarily because of impaired cortical expansion (Fig. $3 H, I$ ).

To determine whether the observed decrease in cortical thickness was because of ZIKV-induced cell death, infected and control tissues were stained for CC3 to mark cells labeled for apoptosis. After 2 DPI of ZIKV exposure, there was a $29.3 \%$ increase in $\mathrm{CC}^{+}$apoptotic cells in the cortical wall, although the total number of apoptotic cells was still very low (Fig. $4 A-C$ ). Ki67 and $\mathrm{PH} 3$ were then used to assess changes in proliferation in infected and control brains. Decreased numbers of $\mathrm{Ki}^{+} 7^{+}$and $\mathrm{PH}^{+}$ cells were found in the VZ of ZIKV-infected neocortex compared with saline-injected littermate controls (Fig. $4 D-I)$. These findings indicate that the fetal ZIKV infection model resulted in increased cell death and a reduction in the number of proliferating NPCs during embryonic neural development. Both disruptions are likely to contribute to the observed microencephaly phenotype.

\section{Temporal infection of major precursor cell types}

To distinguish between apical and basal neocortical precursor cells, we used both immunophenotyping and genetic fate mapping approaches. T-box brain protein 2 (Tbr2) was used as a cell lineage-specific promoter in combination with a Cre-recombinase-dependent dual color reporter. bIPCs were labeled with mCherry fluorescent protein, and non-Tbr2 lineage cells were labeled with ZsGreen fluorescent protein as previously shown by Tyler and Haydar (2013; Fig. 5A). In order to verify the results, 

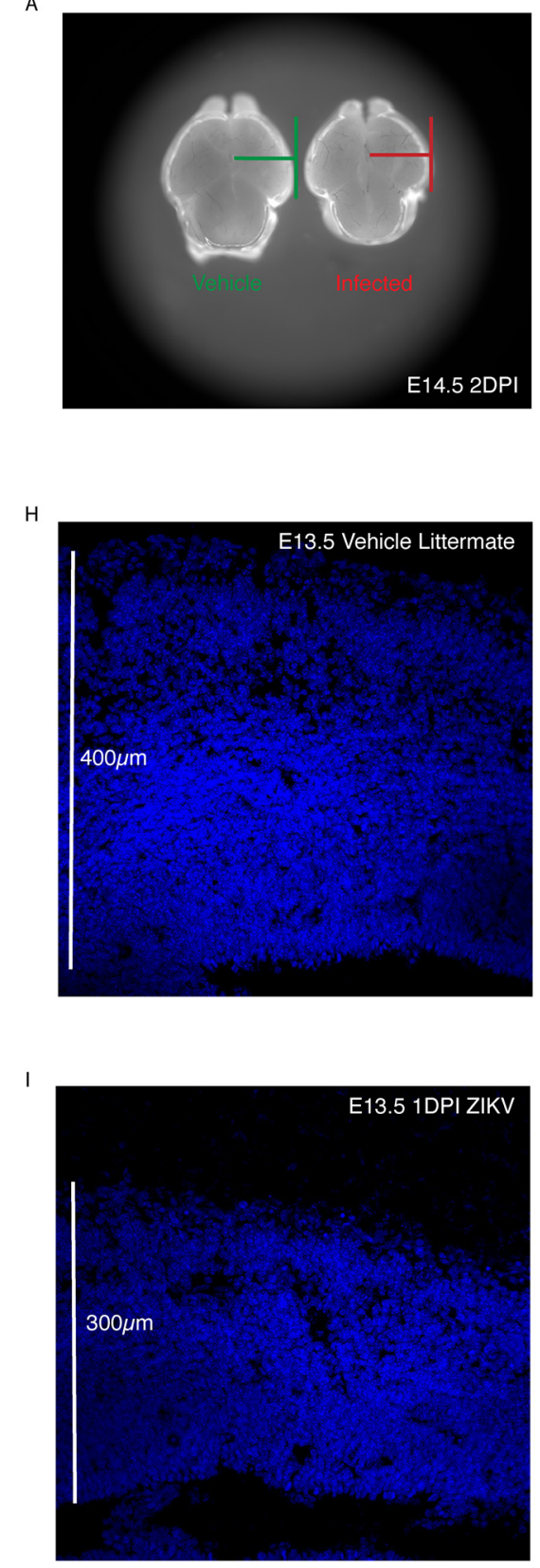

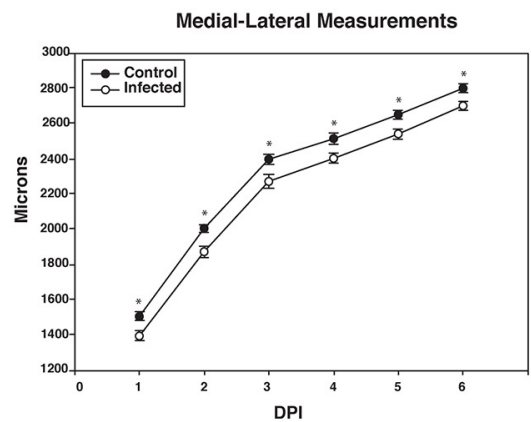

D

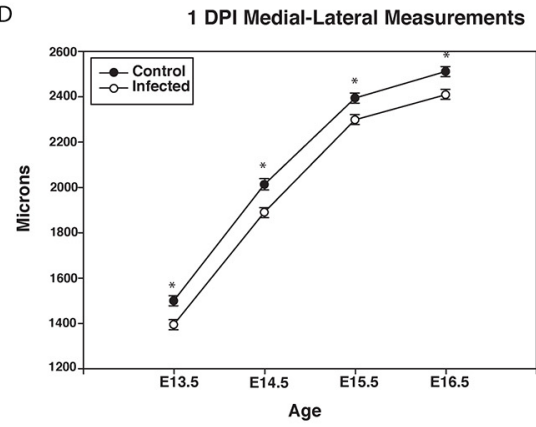

F

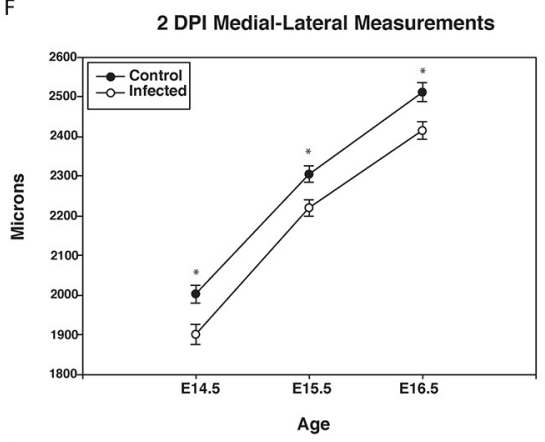

$J$

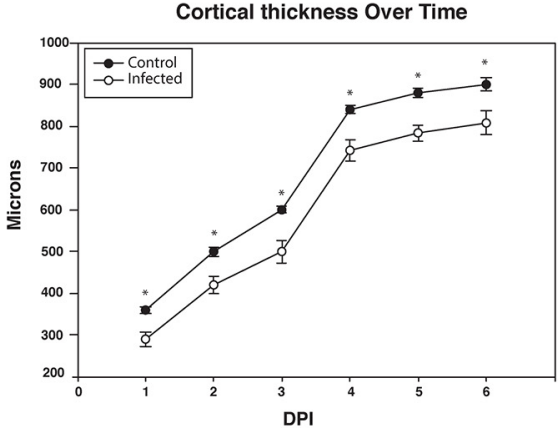

C
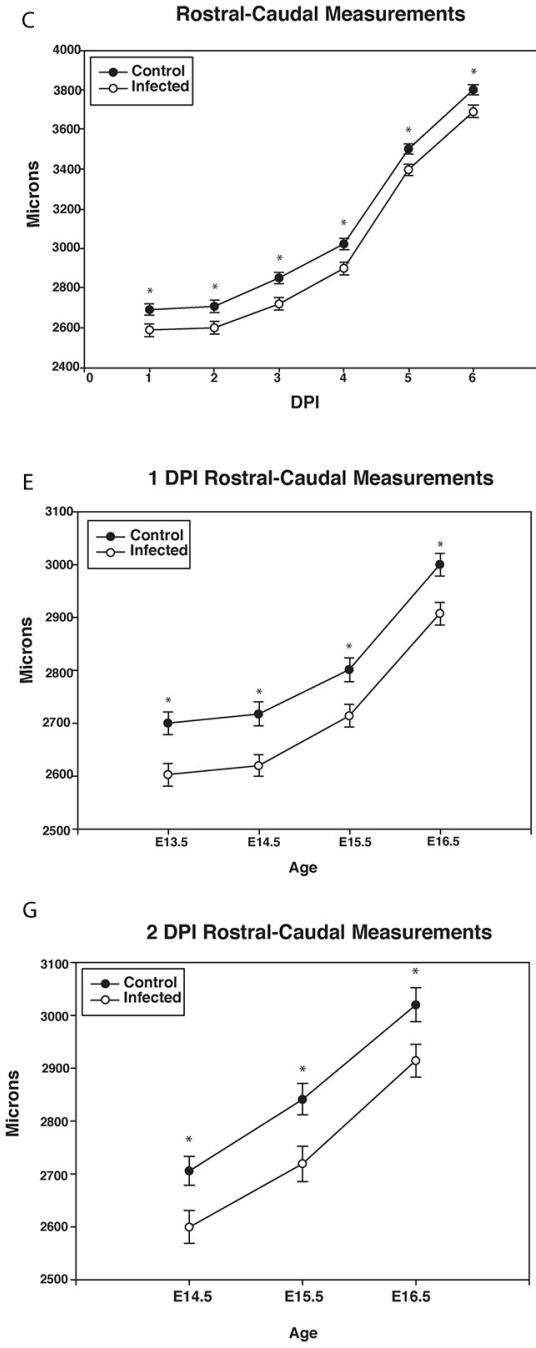

Figure 3. ZIKV decreased forebrain growth regardless of age of infection. Litters were injected at E12.5, E13.5, E14.5, and E15.5 and collected $24 \mathrm{~h}$ later. $\boldsymbol{A}$, Micrographs of example vehicle and ZIKV-infected brain from litter-matched embryos at E14.5 (2 DPI). Medial-lateral $(\boldsymbol{B})$ and rostral-caudal $(\boldsymbol{C})$ brain measurements were significantly decreased after ZIKV infection starting at 1 DPI. The microencephaly phenotype was not dependent on developmental age in $(\boldsymbol{D})$ medial-lateral measurements at $1 \mathrm{DPI}$ or $(\boldsymbol{E})$ rostral-caudal measurements. Brains injected at E12.5, E13.5, and E14.5 and collected $48 \mathrm{~h}$ later also showed the same microencephaly phenotype in $(\boldsymbol{F})$ medial-lateral and $(\boldsymbol{G})$ rostral-caudal measurements. $\boldsymbol{H}$, Representative image of saline injected control neocortical wall collected at E13.5. I, Image of ZIKV injected neocortical wall from the contralateral uterine horn collected at E13.5 (1 DPI). J, Cortical thickness (micrometers from ventricle to pial surface) in $20 \times$ images of coronal brain sections. Control and infected brains were injected at E12.5 and collected 1-6 DPI. Statistical significance was calculated using unpaired $t$ test $\left({ }^{*} p<0.05\right)$ and error bars represent SE. An $n$ of 4 was used for each condition. 
A
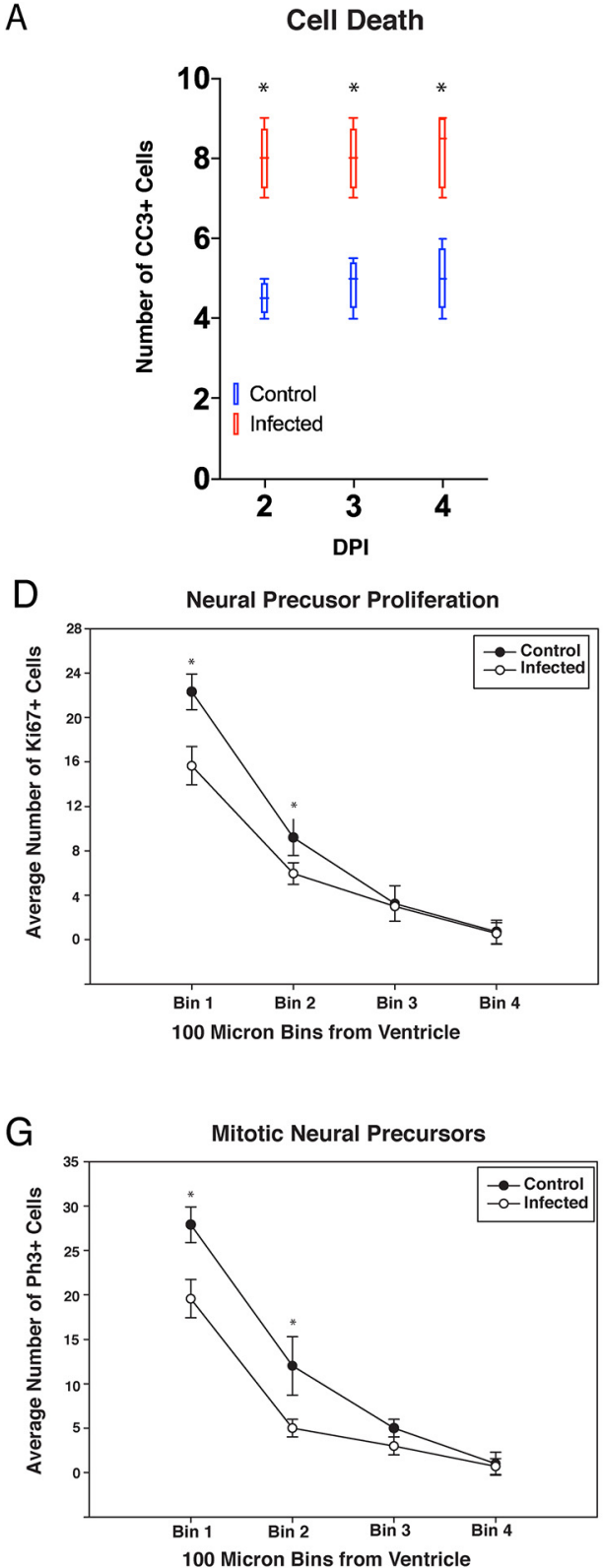
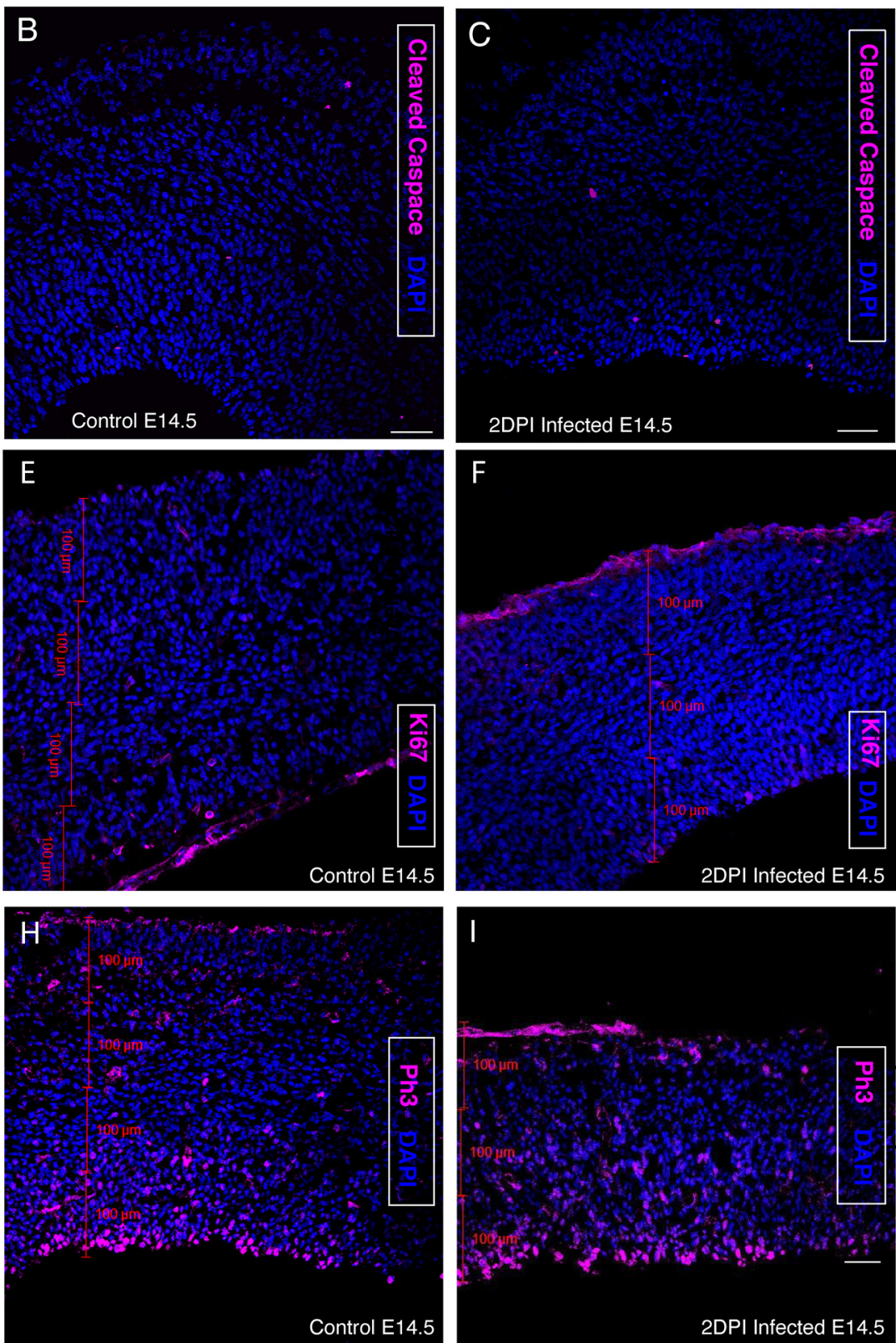

Figure 4. ZIKV infection increased cell death and reduced proliferation in the telencephalic germinal zones. $\boldsymbol{A}$, CC3 ${ }^{+}$cells counted in the neocortical wall of the future somatosensory cortex in vehicle $(n=4)$ and infected $(n=4)$ tissue in 2 DPI (E14.5), 3 DPI (E15.5), and 4 DPI (E16.5) tissue. Statistical significance was calculated using unpaired $t$ test $\left({ }^{*} p<0.05\right)$ and error bars represent SE. B, E14.5 ZIKV-infected (2 DPI) cortical wall stained with CC3. C, E14.5 vehicle control cortical wall stained with CC3. D, Ki67+ average cell number counted in 100- $\mu \mathrm{m}$ bins (bin 1 closest to ventricle, bin 4 closest to pia) in embryos injected at E12.5 and collected at E14.5. Bins 1 and 2, which overlap most with the VZ and SVZ, have significant decreases in proliferating cells following ZIKV infection. $\boldsymbol{E}$, Representative single plane image of control on left and $(\boldsymbol{F})$ infected on right. $\boldsymbol{G}, \mathrm{PH} 3+$ average cell number by bin. Bins 1 and 2, which overlap most with the VZ and SVZ, have significant decreases in proliferating cells following ZIKV infection. $\boldsymbol{H}$, Representative single plane image of control on left and $(I)$ infected on right $(n=4,4)$. Cell numbers were counted in each single plane image of $20-\mu \mathrm{m}$ z-stack images and summed for each bin. Statistical significance was calculated using unpaired $t$ test $\left({ }^{*} p<0.05\right)$ and error bars represent SE.

we measured the compatibility of direct ZIKV infection with IUE labeling. We compared levels of viral infection and plasmid transfection between ZIKV, plasmid DNA, and a combination of the two. This assessed whether infectibility and the efficiency of these plasmid reporters were compromised by the combined approach. To account for variations in fetal development when comparing NPCs in infected and control mice, one uterine horn was injected with ZIKV/plasmid cocktail and the other was injected with plasmid cocktail and/or saline. IHC (Fig. 5B-F) 

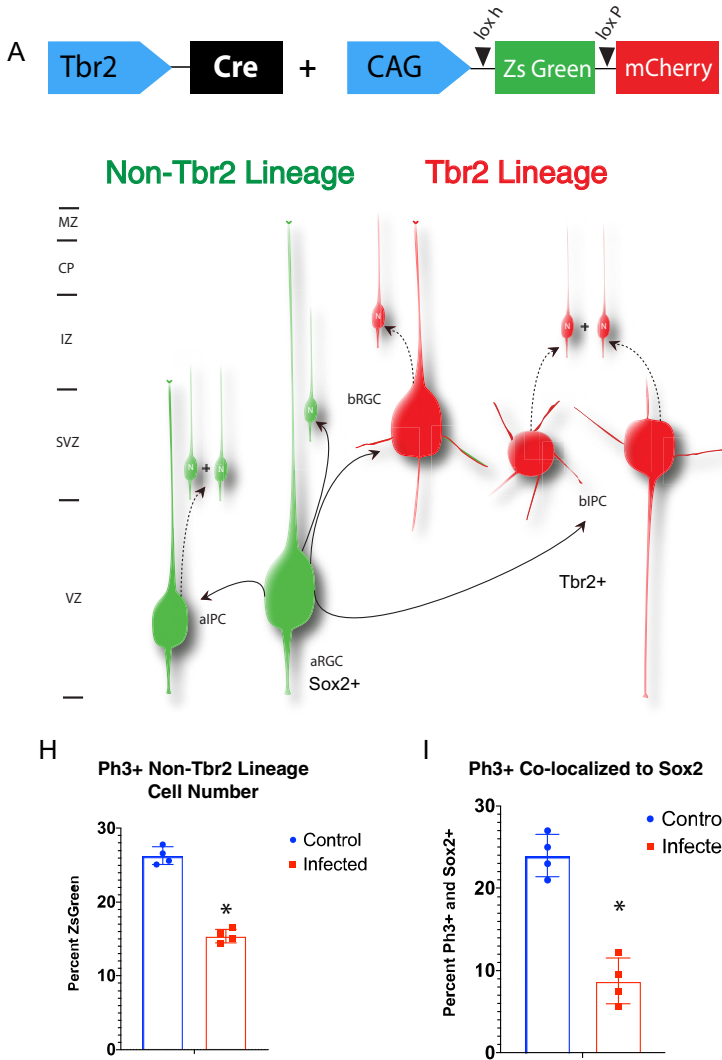

J

Ph3+Tbr2 Lineage Cell Number

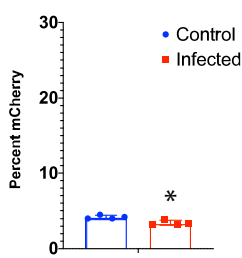

Percent Ph3 Co-localized to Tbr2

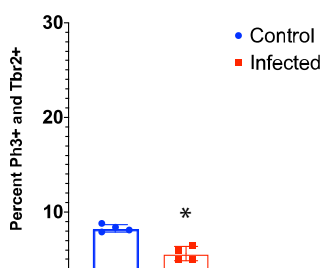

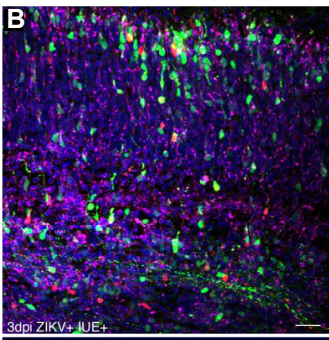
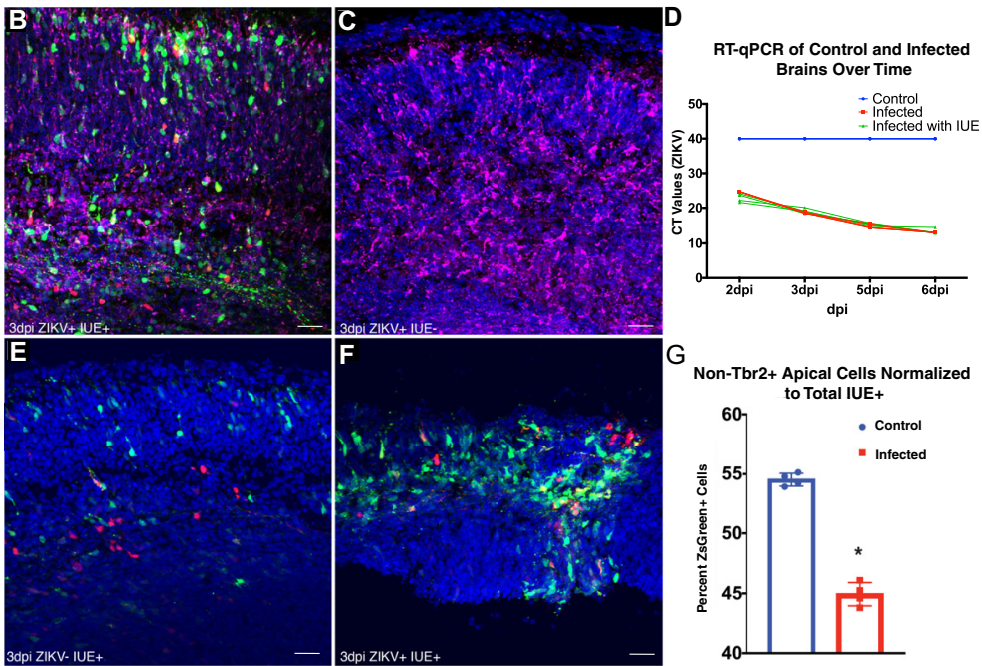

$\mathrm{L}$
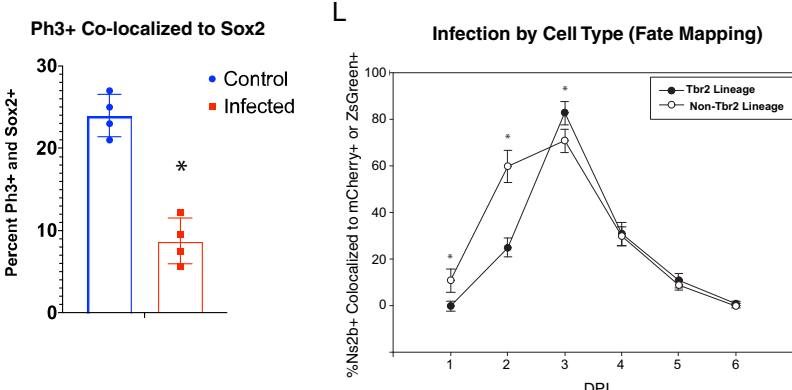

$\mathrm{M}$

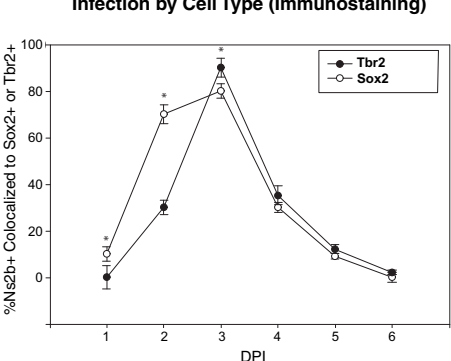

Figure 5. ZIKV causes a decrease in fate mapped apical precursor cell number. $\boldsymbol{A}$, Somatosensory cortex in control and infected brains was transfected with Tbr2-Cre + CAG-StopLight at E13.5 and collected $48 \mathrm{~h}$ later (E15.5), labeling the apical progenitors in green and the basal Tbr2 lineage progenitors in red. B, 3 DPI (E15.5) infected tissue with and without (C) IUE show no difference in NS2B labeling. Scale bars: $50 \mu \mathrm{m}$. $\boldsymbol{D}$, ZIKV RT-qPCR in IUE and non-IUE-infected brains across time show no difference in infection. $\boldsymbol{E}$, Vehicle control electroporated with Tbr2-Cre and CAG-StopLight. Scale bar: $50 \mu \mathrm{m}$. F, 3 DPI (E15.5) infected brain electroporated with Tbr2-Cre and CAG-StopLight. Scale bar: $50 \mu \mathrm{m}$. G. The percentage of ZsGreen ${ }^{+}$cells in the total electroporated population were reduced in infected tissue compared with control $(n=4,4)$. Statistical significance was calculated using unpaired $t$ test $\left({ }^{*} p<0.05\right)$ and error bars represent SE. $\boldsymbol{H}$, Tbr2-Cre CAG-SL electroporated brains were collected after $48 \mathrm{~h}$ (IUE on E12.5). Control and infected brain sections were stained for PH3. The number of $\mathrm{PH}^{+}{ }^{+} \mathrm{Tbr} 2$ lineage $\left(\mathrm{mCherry}^{+}\right)$cells was decreased by $\mathrm{ZIKV}$ infection. I, The number of $\mathrm{PH}^{+}$non-Tbr2 lineage $\left(\mathrm{ZsGreen}^{+}\right)$cells were decreased by ZIKV infection. E14.5 control and infected brain sections were immunostained for $\mathrm{PH} 3$ and Tbr2. $\boldsymbol{J}$, The number of $\mathrm{PH}^{+}$, Tbr2 ${ }^{+}$cells were decreased by $\mathrm{ZIKV}$ infection. $\boldsymbol{K}$, The number of $\mathrm{PH}^{+}$, Sox ${ }^{+}$cells was substantially decreased by ZIKV infection. Statistical significance was calculated using unpaired $t$ test $\left({ }^{*} p<0.05\right)$ and error bars represent SE. A sample size of four was used in each condition. $L$, Tbr2-StopLight fate mapping identifies temporal sequence of cell type infection. Brains were electroporated and co-infected with ZIKV at E12.5 and collected 1-6 DPI. Cell counts from confocal z-stacks indicate that ZsGreen ${ }^{+}$apical precursors are preferentially infected at 1 and 2 DPI. At 3 DPI, more mCherry ${ }^{+}$basal precursors are infected than apical precursors. Thereafter, infection decreased in both cell types $(n=4$, 4). Statistical significance was calculated using unpaired $t$ test $\left({ }^{*} p<0.05\right)$ and error bars represent SE. M, Immunohistochemical staining for apical (Sox2) and basal (Tbr2) precursors demonstrates temporal spread of infection through the germinal zones. Brains were injected with ZIKV at E12.5 and collected 1-6 DPI. Results indicate that Sox $2^{+}$apical precursors are preferentially infected at 1 and 2 DPI. At $3 \mathrm{DPI}$, more Tbr2 ${ }^{+}$basal precursors are infected. In later time points, both cell types are infected equally $(n=4,4)$. Statistical significance was calculated using unpaired $t$ test $\left({ }^{*} p<0.05\right)$ and error bars represent SE. 
and RT-qPCR (Fig. 5D) demonstrated no crossover of virus from the infected side to the control side or into the dam's blood. The validity of this approach was confirmed as it resulted in the same plasmid transfection efficiency and ZIKV infection dynamics when ZIKV and IUE were conducted simultaneously. This allowed for the analysis of red and green cell numbers in control and infected tissue. At $3 \mathrm{DPI}$, infected tissue had significantly decreased numbers of ZsGreen ${ }^{+}$cells, suggesting that the apical population of NPCs may be preferentially targeted by ZIKV (Fig. 5G). To assess whether ZIKV affected the proliferative capabilities of specific cell types, E12.5 embryos were infected with ZIKV and electroporated with Tbr2-Cre and CAG-StopLight before collection $48 \mathrm{~h}$ later. We found an $18.2 \%$ (Fig. $5 H$ ) decrease in mCherry $/ \mathrm{Ph}^{+}$and a $40 \%$ decrease in the number of $\mathrm{ZsGreen}^{+} / \mathrm{Ph}^{+}$co-labeled cells in infected tissue compared with controls (Fig. 5/). To confirm that aRGCs were more affected than bIPCs at 2 $\mathrm{DPI}$, non-electroporated ZIKV-infected brain samples were immunostained for Sox2 and Tbr2, marking aRGCs and bIPCs respectively. While both cell types showed decreased numbers of $\mathrm{Ph}^{+}$cells at $2 \mathrm{DPI}$, Sox ${ }^{+}$cells were particularly impacted with a $60 \%$ decrease compared with the $28.6 \%$ decrease found in $\mathrm{Ph}^{+} / \mathrm{Tbr}^{+}$cells (Fig. $5 J, K)$. These experiments confirmed that aRGCs are targeted by ZIKV early in infection and that proliferation of this essential NPC class is reduced.

\section{Precursor type consequences of persistent ZIKV infection}

Since aRGCs are particularly targeted in acute infection, we next sought to understand how longer infection times affect neocortical growth. Tbr2-Cre and CAG-StopLight electroporated tissue was stained with NS2B in control and infected tissue at 1 DPI through 6 DPI. ZsGreen ${ }^{+} / \mathrm{NS} \mathrm{B}^{+}$and $\mathrm{mCherry}^{+} / \mathrm{NS} \mathrm{B}^{+}$populations were counted on each day, and all cell counts were normalized to the total number of electroporated cells to account for the normal variability in the size of the electroporated field. Increased numbers of ZsGreen infected cells $\left(\mathrm{ZsGreen}{ }^{+} / \mathrm{NS} \mathrm{B}^{+}\right)$were found at 1 and $2 \mathrm{DPI}$. However, at $3 \mathrm{DPI}$, the number of infected mCherry cells $\left(\mathrm{mCherry}^{+} / \mathrm{NS} \mathrm{B}^{+}\right.$) surpassed the number of infected ZsGreen cells. The proportion of infected mCherry and ZsGreen cells equalized at 4-6 DPI as fewer NPCs were $\mathrm{ZIKV}^{+}$over time (Fig. $5 L, M$ ). To further confirm cell type vulnerability, non-electroporated tissue was immunostained for Sox2 and Tbr2 at the same time points. Again, NS2B co-localized with more Sox $2^{+}$cells at 1 and 2 DPI. At 3 DPI, increased numbers of Tbr2 ${ }^{+}$mCherry cells co-localized with NS2B (Fig. 7B). These findings suggest that aRGCs are initially targeted by ZIKV and that aRGC-derived bIPCs gain active ZIKV infection either from cytoplasmic division or from virions shed from infected aRGCs.

\section{ZIKV infection alters neocortical cytoarchitecture}

The labeling of aRGCs and bIPCs with the dual color fluorescent protein reporter allowed for visualization of changes in whole cell morphology in response to ZIKV infection. In particular, we found noticeable patterns of disorganization in the basal processes of aRGCs that stretch to the pial surface from the cell bodies within the VZ.
aRGC basal fibers are required for neuronal migration during development and may elucidate the mechanism behind decreased neuronal migration after infection. Rosenfeld et al. (2017) described perturbed cytoarchitecture after ZIKV infection using organotypic mouse brain slice cultures electroporated with CAG-GFP but particular changes to the aRGC palisade have not yet been quantified. To test whether the number of basal fibers had decreased after infection, we counted the number of end feet reaching the pial surface in control and infected tissue at $2 \mathrm{DPI}$, normalized to the number of IUE ${ }^{+}$cells present. While a decrease in aRGC fibers/endfeet may be because of the decrease in aRGC numbers after infection, the above fate mapping studies identified only an $18.2 \%$ decrease in apical ZsGreen ${ }^{+}$cells at 2 DPI (Fig. 5G). In contrast, endfeet counts denote a $60 \%$ decrease in aRGC basal fibers at this same time point, indicating that the interrupted basal process phenotype is more pronounced than the decrease in numbers of aRGCs (Fig. $6 A, B$ ). Loss or retraction of aRGC basal fibers from the superficial cortical layers may lead to neuronal migration defects and ultimately cause decreased cortical thickness and hypoplasia.

To confirm the loss of aRGC basal processes, the lipophilic dye Dil was applied to the exterior surface of control and infected brains after the removal of the meninges. Allowing two weeks for passive intramembranous diffusion, we found that the density of Dil labeling was decreased in the VZ and SVZ of infected tissue. While neural projections and somata were Dil labeled in the cortical plate of infected tissue, the aRGC basal fibers were sparse in brains exposed to ZIKV compared with vehicle control brains (Fig. $6 C-G$ ). To quantify this difference, the Dil intensity was measured in the VZ and in the area of greatest fluorescence directly above the VZ in the intermediate zone (IZ). For each image, the VZ intensity was normalized to the $I Z$ intensity to account for differences in Dil penetration between sections and brains. Infected tissue showed a $\sim 70 \%$ reduction in Dil intensity (Fig. $6 G$ ). Together, the IUE and Dil studies demonstrate that ZIKV infection causes disruption to the neocortical cytoarchitecture by disrupting aRGC basal processes.

\section{aRGC heterogeneity}

In contrast to longstanding assumptions, recent data indicate that the aRGC population is heterogeneous and can undergo temporal transcriptomic change during the neurogenesis period (Miyata et al., 2004; Tyler et al., 2015; Heavner et al., 2020). In ZIKV-infected tissue, we found that a proportion of aRGC fibers were perturbed while others appeared to develop normally. This suggested that there may be intrinsic differences between these affected and non-affected cells that may stem from underlying aRGC heterogeneity. To test whether subpopulations of aRGCs may be differentially susceptible to infection by ZIKV, we took advantage of a recent scRNAseq study in the E15.5 mouse neocortex (Li et al., 2020). This dataset reveals two distinct clusters of cortical aRGCs (aRGC1 and aRGC2). We sought to determine whether genes differentially expressed between these two groups 


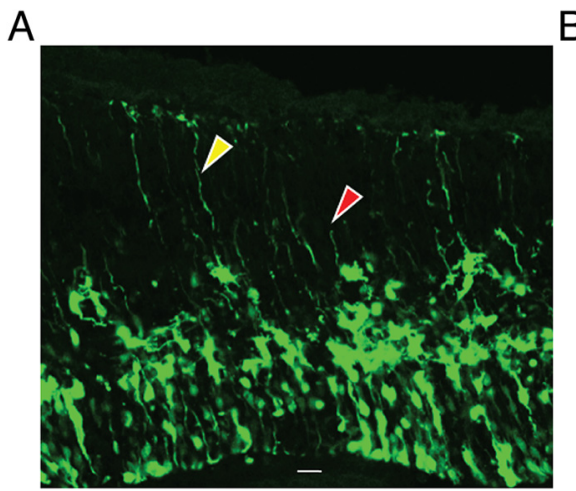

B
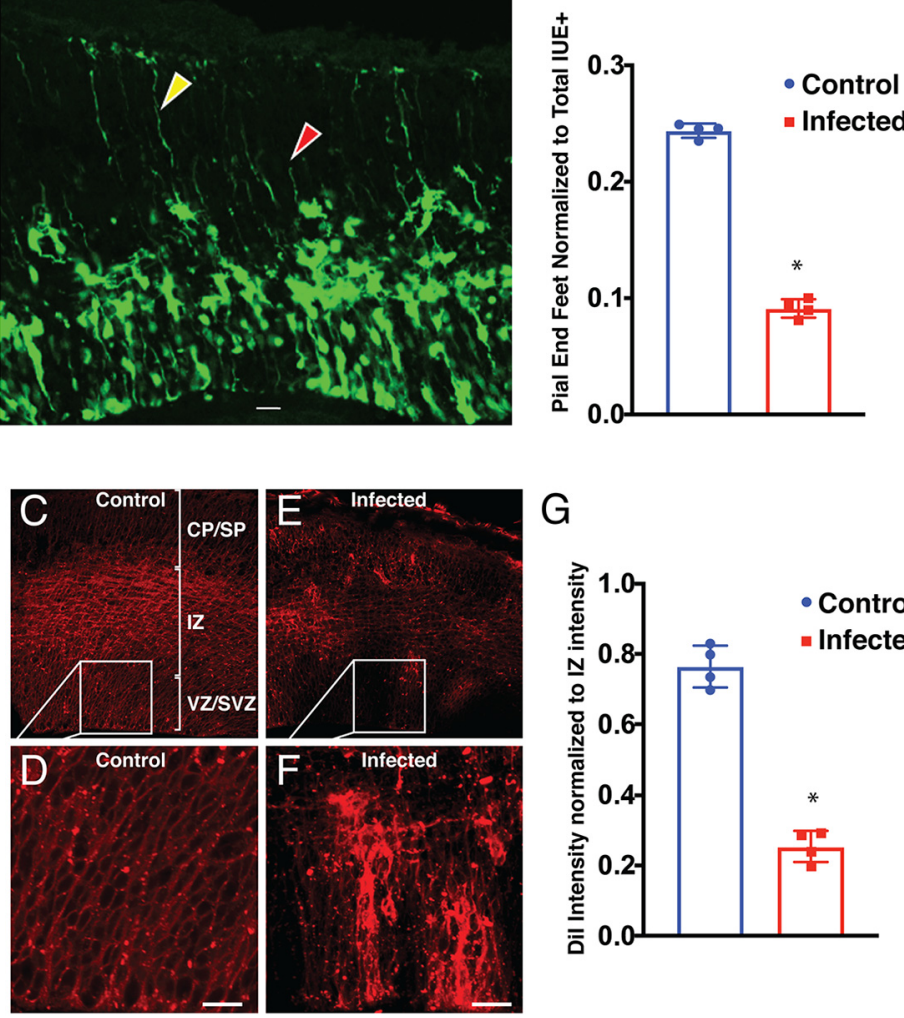

Figure 6. Forebrain ZIKV infection disrupts radial scaffolding of the telencephalic wall. Brains were electroporated with Tbr2-Cre + CAG-StopLight and either vehicle or ZIKV at E13.5 and collected $48 \mathrm{~h}$ later (E15.5). A, ZIKV disrupts cytoarchitecture. Example of maximum projection z-stack of electroporated infected tissue at 2 DPI, showing normally developing (yellow arrow) and perturbed basal processes (red arrow). Scale bar: $20 \mu \mathrm{m}$. $\boldsymbol{B}$, Basal fiber end foot number is decreased in infected tissue. Cells were counted in the electroporated region of somatosensory cortex in 3D image stacks. The number of end feet was normalized to IUE ${ }^{+}$cell number and was significantly decreased in infected tissue $(n=4,4)$. Statistical significance was calculated using unpaired $t$ test $\left({ }^{*} p<0.05\right)$ and error bars represent SE. Dil was applied to the surface of E15.5, 2 DPI control and infected littermates and was allowed to diffuse for two weeks at room temperature. $\boldsymbol{C}$, Dil applied to the pial surface of the cortex labeled cell membranes in the VZ by diffusion down basal fibers. Dil labeling in the vehicle-injected cortex shows complete labeling of aRGC palisade from the pial to the ventricular surface. $\boldsymbol{D}$, Inset from $\boldsymbol{C}$ illustrates normal Dil intensity. Scale bar: $20 \mu \mathrm{m}$. $\boldsymbol{E}$, Basal fibers are disrupted in infected tissue. Dil labeling in 2 DPI-infected cortex reveals many areas of neocortical wall with patchy diffusion; somata and neurites are labeled in the $I Z$ and CP but very little Dil label is found in the VZ and SVZ. $\boldsymbol{F}$, Inset from $\boldsymbol{E}$ illustrates sparse labeling of fibers in the VZ and SVZ of 2 DPI cortex. Scale bar: $20 \mu \mathrm{m}$. G, Dil intensity in VZ/SVZ was normalized to the brightest intensity in the IZ directly above measurement taken in the VZ/SVZ. The VZ/SVZ intensity was significantly decreased in infected tissue $(n=4,4)$. Statistical significance was calculated using unpaired $t$ test $\left({ }^{*} p<0.05\right)$ and error bars represent SE.

may identify separate populations of aRGCs with differential vulnerability to ZIKV infection.

To develop markers for these two clusters of aRGCs, differential gene expression in aRGC1 and aRGC2, compared with gene expression profiles of other NPC and

Table 1: Group 1 and group 2 aRGC markers chosen from scRNAseq data

\begin{tabular}{lll}
\hline Group & Fold change & $p$ value \\
\hline Group 1, Prc1 & 6.3 & $5.60 \mathrm{E}-35$ \\
Group 1, AxI & 2.3 & $9.87 \mathrm{E}-03$ \\
Group 2, MFAP2 & 0.64 & $1.54 \mathrm{E}-08$ \\
Group 2, Robo4 & 6.2 & $1.30 \mathrm{E}-32$
\end{tabular}

The fold change represents differential expression between aRGC1 and aRGC2. For details, see Materials and Methods. neuron clusters, was used to create a list of potential transcriptional markers specific to aRGC1 cells or to aRGC2 cells. Prc1 and $A X L$ were top candidate genes for aRGC1 and MFAP2 and Robo4 were top candidate genes for aRGC2 (Table 1). Conserved regulatory regions proximal to the transcriptional start sites of these candidate genes were used to construct Cre-driver plasmids, which were tested by IUE using the CAG-StopLight reporter plasmid at 24 and $48 \mathrm{~h}$. This approach yielded mCherry ${ }^{+}$recombinant cells in the VZ with the expected morphology and location of aRGCs (Fig. 7A-C). Prc1-Cre labeled a larger proportion of cells compared with MFAP2-Cre (7D-E). $\mathrm{PrC}^{+}$cells also showed greater proliferative potential compared with $M F A P 2^{+}$cells as seen in the proportion of mCherry ${ }^{+}$cells counted between 24 and $48 \mathrm{~h}$ (Fig. $7 l, J)$. 


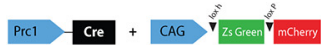
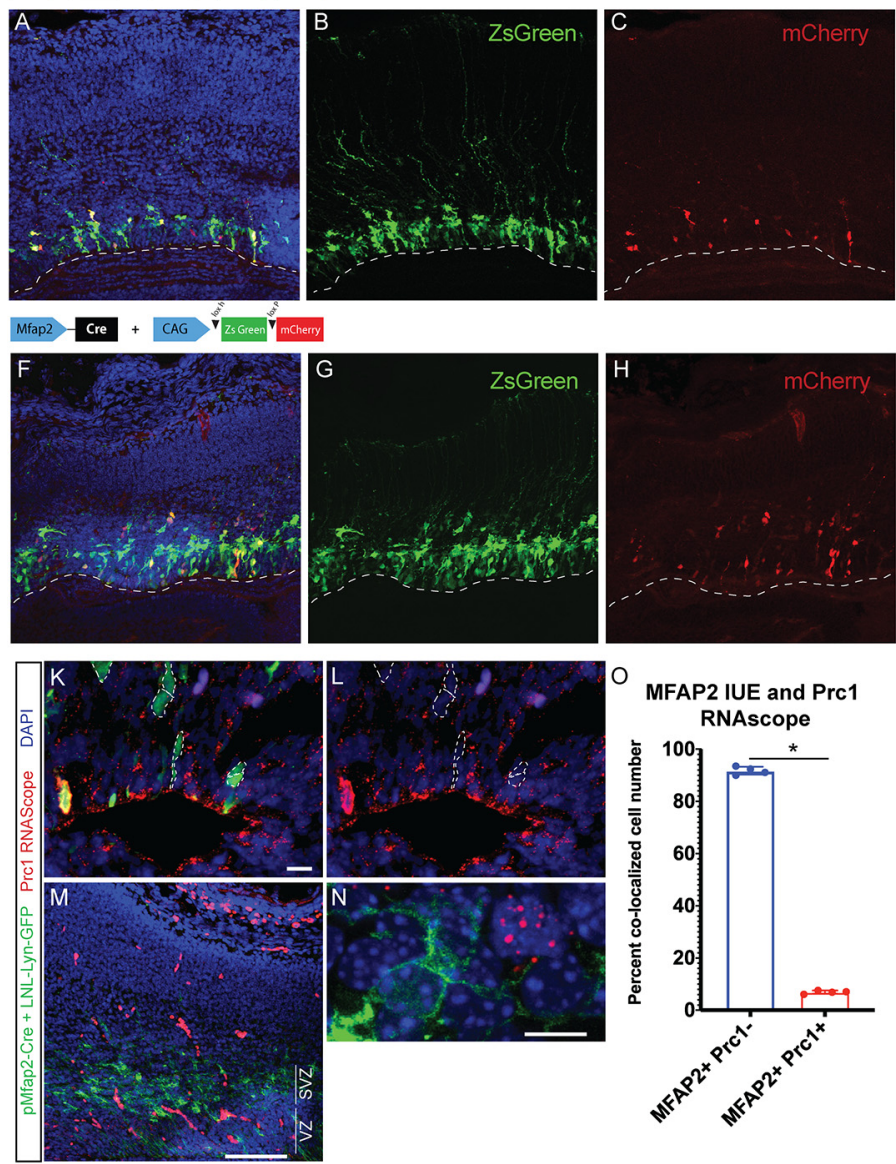

D Prc1-Cre CAG-StopLight 24hrs E Prc1-Cre CAG-StopLight 48hrs
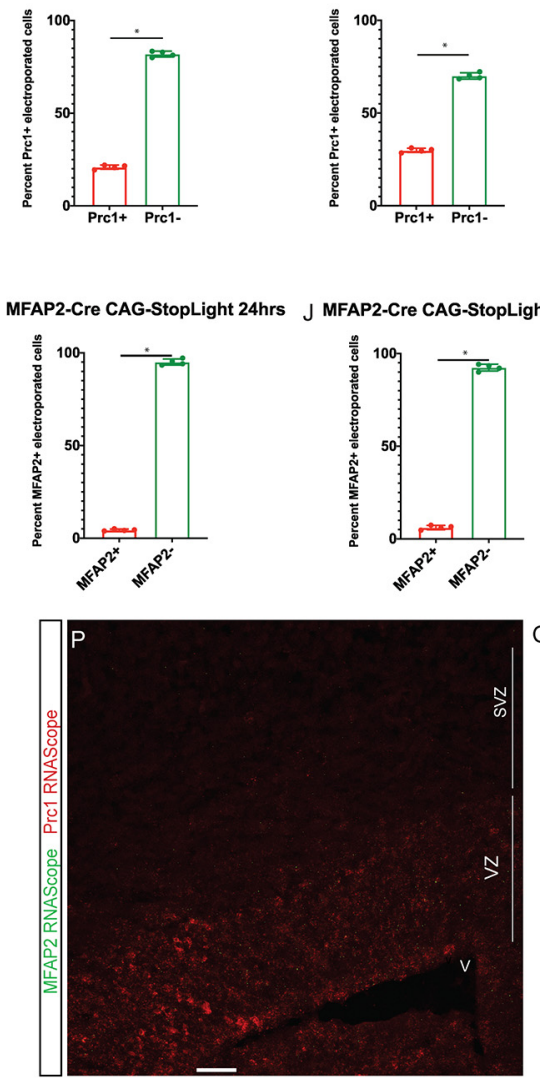

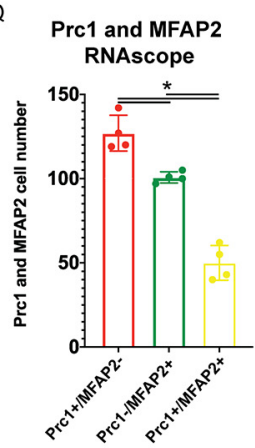

Figure 7. Fate mapping of aRGC types. E14.5 mice were electroporated with Prc1-Cre or MFAP2-Cre and CAG-StopLight and collected 24 or 48 h later. A, IUE with Prc1-Cre and CAG-StopLight produced VZ-localized mCherry ${ }^{+}$cells. A composite image from Prc1-Cre experiments with non-specific electroporated cells in green $(\boldsymbol{B})$ and $(\boldsymbol{C}) \operatorname{Prc} 1^{+}$cells in red. Scale bar: $50 \mu \mathrm{m}$. Red and green cell number were counted and normalized to the total number of electroporated cells for $(\boldsymbol{D})$ 24-h and $(\boldsymbol{E})$ 48-h time points. $\boldsymbol{F}$, IUE with MFAP2-Cre and CAG-StopLight produced VZ localized mCherry ${ }^{+}$cells. A composite image from MFAP2-Cre and CAG-SL recombination allowing visualization of electroporated cells in green $(\boldsymbol{G})$ and $(\boldsymbol{H}) M F A P 2^{+}$cells in red. Scale bar: $50 \mu \mathrm{m}$. Red and green cell number were counted and normalized to the total number of electroporated cells for $24-\mathrm{h}(\boldsymbol{I})$ and $48-\mathrm{h}(\boldsymbol{J})$ time points $(n=4$ per condition). Statistical significance was calculated using unpaired $t$ test $\left({ }^{*} p<0.05\right)$ and error bars represent SE. $K$, E13.5 mice were electroporated with MFAP2-Cre and CAGLNL-LYN-GFP and collected $48 \mathrm{~h}$ later. Prc $1^{+}$cells were labeled with RNAscope (red), electroporated MFAP2+ cells with GFP and nuclei were labeled with Hoechst (blue). Scale bar: $15 \mu \mathrm{m}$. $\boldsymbol{L}$, Prc1 RNAscope alone. $\boldsymbol{M}, 20 \times$ magnification image with no zoom shows marker labeling in the VZ and SVZ $48 \mathrm{~h}$ after electroporation. Scale bar: $100 \mu \mathrm{m}$. N, Zoom of a Prc1 ${ }^{+}$cell in red neighboring a $\mathrm{MFAP2}^{+}$in green. Scale bar: $10 \mu \mathrm{m}$. $\mathbf{O}$, Cells counted positive for RNAscope by including two or more puncta within the cell body that were co-localized with GFP from electroporation were normalized to the total number of electroporated cells in the field to determine percent co-localized cell number. Seven percent of the electroporated cells were positive for Prc1 RNAscope $(n=4)$. Statistical significance was calculated using unpaired $t$ test $\left({ }^{*} p<0.05\right)$ and error bars represent SE. $\boldsymbol{P}$, E15.5 cryosectioned tissue was stained for MFAP2 (green), and Prc1 (red) using RNAscope. Scale bar: $50 \mu \mathrm{m}$. Q, Prc $1^{+}$cells, MFAP2 ${ }^{+}$cells, and co-labeled cells were counted in $20-\mu \mathrm{m}$ z-stack images $(n=4)$. Statistical significance was calculated using a one-way ANOVA $\left({ }^{*} p<0.05\right)$ and error bars represent SE.

The candidate marker genes for each cortical aRGC type were also used as targets for multiplexed single molecule in situ hybridization. Prc1 RNAscope hybridization was performed on 24 h MFAP2-Cre + CAG-LNL-LynGFP electroporated tissue to determine whether these markers indeed label different aRGCs. It was found that only $7 \%$ of the $M F A P 2^{+}$electroporated cells were also positive for Prc1 transcript (Fig. 7K-O). Using multiplexed hybridization of PrC1 and MFAP2 RNAscope probes recapitulated results showing that there is separation of marker expression in these cell types and that there are more Prc1+ only cells followed by MFAP2 ${ }^{+}$only cells, with the smallest proportion being $P r C 1^{+} / M F A P 2^{+}$cells (Fig. 7P,Q).

Given the separation of $\operatorname{Prc}^{+}$and $M F A P 2^{+}$populations, we next tested whether these groups were differentially vulnerable to ZIKV infection by multiplexing RNAscope probes against Prc1, MFAP2, and ZIKV. ZIKV was found to be colabeled in $\mathrm{PrC}^{+}$cells significantly more often than in $\mathrm{MFAP}^{+}$cells. In fact, at $2 \mathrm{DPI}, 63 \%$ of $\mathrm{Prc}^{+}$cells were ZIKV-infected while only $35 \%$ of $M F A P 2^{+}$cells were infected (Fig. 8A,B). 


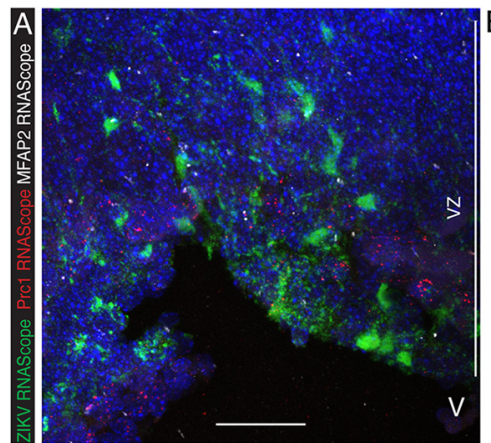

${ }^{B}$ ZIKV Infection by aRGC Type
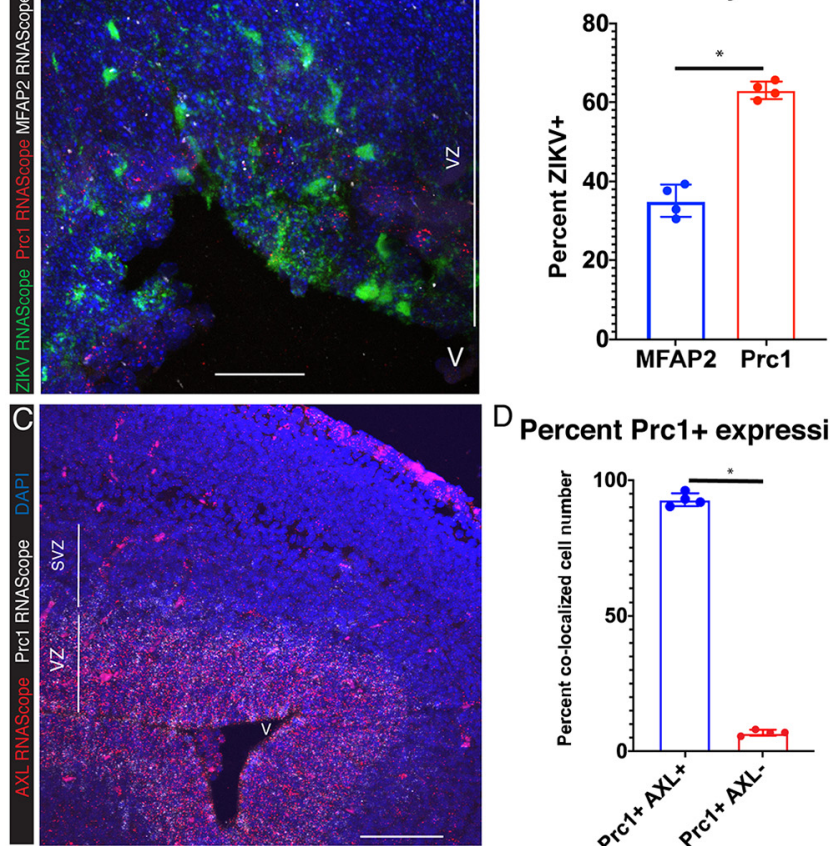

${ }^{D}$ Percent Prc1+ expressing AXL
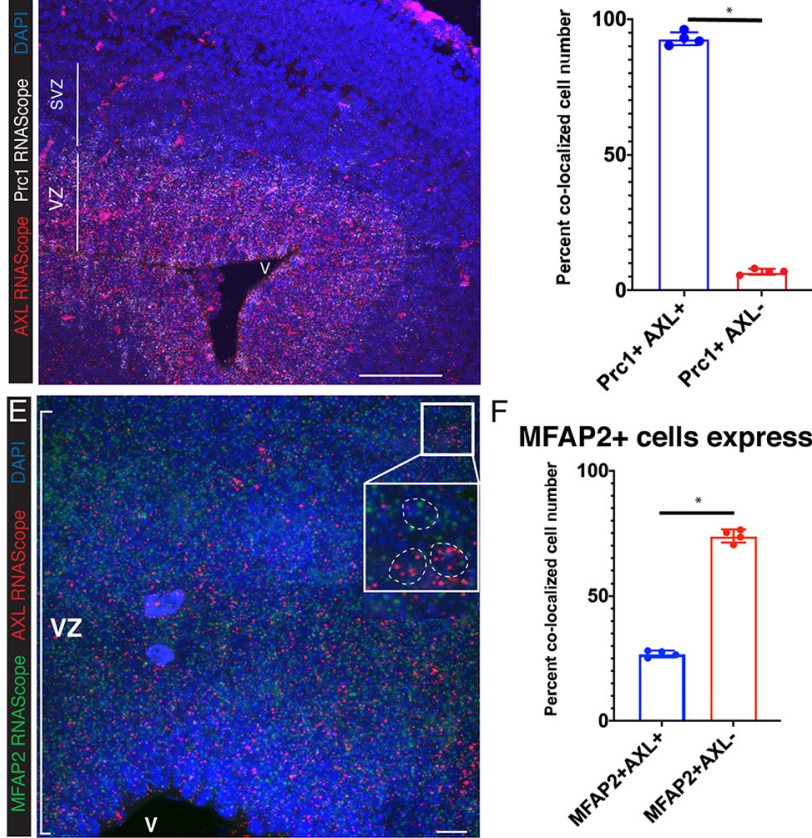

MFAP2+ cells expressing AXL
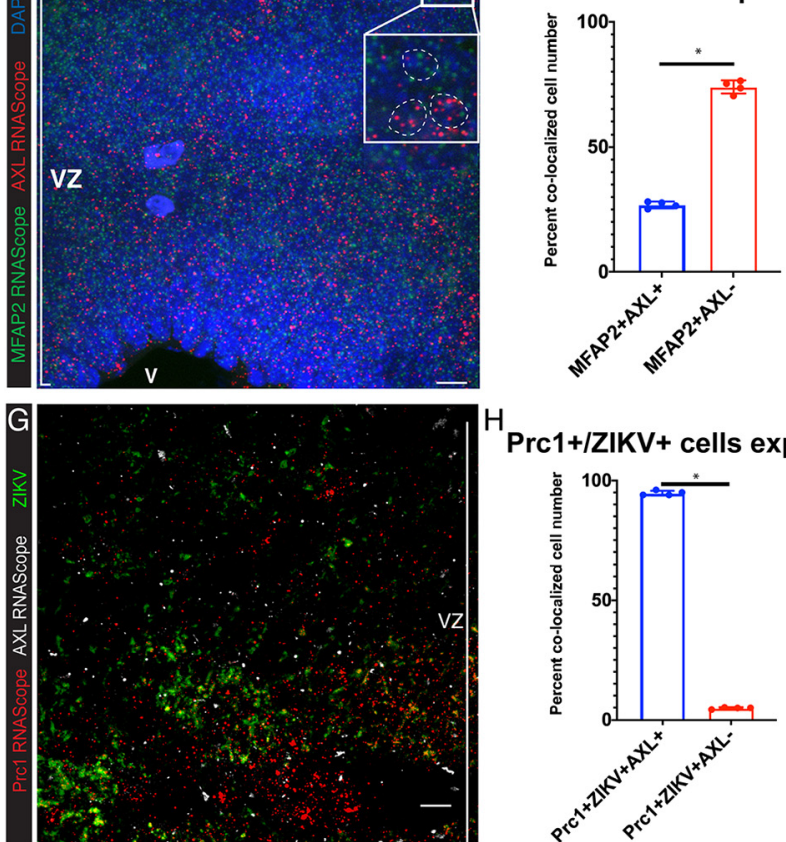

${ }^{H}$ Prc1+/ZIKV+ cells expressing AXL

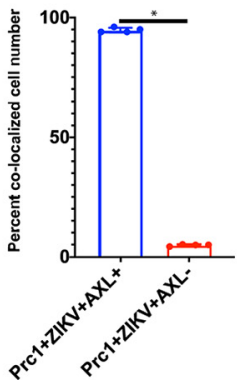

Figure 8. $\mathrm{MFAP2}^{+}$aRGCs show lower infection rates compared with Prc ${ }^{+}$aRGCs. $\boldsymbol{A}$, Multiplexed RNAscope staining shows fewer infected MFAP2 ${ }^{+}$cells compared with $P r c 1^{+}$cells. E15.5 2 DPI infected tissue was stained for ZIKV (green), Prc1 (red), and MFAP2 (white) using RNAscope. Scale bar: $50 \mu \mathrm{m}$. B, Prc $1^{+}$cells are more vulnerable to ZIKV infection than MFAP2 ${ }^{+}$cells. Statistical significance was calculated using unpaired $t$ test $\left({ }^{*} p<0.05\right)$ and error bars represent SE $(n=4)$. $\boldsymbol{C}, \operatorname{Prc} 1$ and $A X L$ are highly expressed in the telencephalic germinal zone. E15.5 tissue was stained for AXL (red) and Prc1 (white) using RNAscope. Scale bar: $80 \mu \mathrm{m}$. D. Over $90 \%$ of Prc1+ cells co-express $A X L(n=4)$. Statistical significance was calculated using unpaired $t$ test $\left({ }^{*} p<0.05\right)$ and error bars represent SE. $\boldsymbol{E}$, The majority of MFAP2 ${ }^{+}$cells are negative for $A X L$ expression. The white box outlines a zoomed example of MFAP2 ${ }^{+}$cells with and without $A X L$ co-expression showing variability of $A X L$ expression in $M F A P 2^{+}$cells. Scale bar: $10 \mu \mathrm{m} . \boldsymbol{F}, A X L$ expression is decreased in $M F A P 2^{+}$cells compared with $P r c 1^{+}$cells. Despite the lower infection rate in group 2 aRGCs, $26.5 \%$ of MFAP2 ${ }^{+}$cells co-expressed $A X L$. G, ZIKV infection correlates with AXL expression in Prc $1^{+}$cells. E15.5 tissue was stained for Prc1 (red) and $A X L$ (white) and ZIKV (green) using RNAscope. Scale bar: $10 \mu \mathrm{m}$. $\boldsymbol{H}$, The majority of $\operatorname{Prc} 1^{+}$, ZIKV ${ }^{+}$cells also express $A X L$. Over $95 \%$ of infected $P r c 1^{+}$cells were co-labeled with $A X L$ RNAscope. Statistical significance was calculated using unpaired $t$ test $\left({ }^{*} p<0.05\right)$ and error bars represent $\mathrm{SE}(n=4)$. 
Table 2: Key resource table

\begin{tabular}{|c|c|c|c|c|}
\hline \multicolumn{5}{|c|}{ Key resources table } \\
\hline Reagent type or resource & Designation & Source or reference & Identifiers & Additional information \\
\hline Sequence-based reagent & Prc1 ISH probe & Advanced Cell Diagnostics & 57712 & MFISH \\
\hline Sequence-based reagent & AXL ISH probe & Advanced Cell Diagnostics & 450931 & MFISH \\
\hline Sequence-based reagent & MFAP2 ISH probe & Advanced Cell Diagnostics & 445421 & MFISH \\
\hline Sequence-based reagent & Robo4 ISH probe & Advanced Cell Diagnostics & 466321 & MFISH \\
\hline Sequence-based reagent & V-ZIKA-06 ISH probe & Advanced Cell Diagnostics & 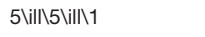 & MFISH \\
\hline Antibody & Ns2b & Genetex & GTX133308 & Anti-rabbit (1:250) \\
\hline Antibody & CC3 & Cell Signaling & 9661-s & Anti-rabbit (1:250) \\
\hline Antibody & Ki67 & BS Pharmigen & MAB1567 & Anti-mouse (1:250) \\
\hline Antibody & $\mathrm{Ph} 3$ & Millipore & $06-570$ & Anti-rabbit (1:300) \\
\hline Antibody & Tbr2 & Millipore, Santa Cruz & AB2283, sc-293481 & Anti-rabbit (1:300), Anti-mouse (1:250) \\
\hline Antibody & Sox2 & Santa Cruz & sc-17320 & Anti-goat (1:250) \\
\hline Antibody & Tuj1 & Santa Cruz & sc-\?\0005 & Anti-mouse (1:250) \\
\hline Secondary & Goat anti-rabbit & Invitrogen & A11008 & Goat anti-rabbit (1:500) \\
\hline Secondary & Goat anti-mouse & Invitrogen & A21050 & Goat anti-mouse (1:500) \\
\hline Secondary & Donkey anti-mouse & Invitrogen & A2202 & Donkey anti-mouse (1:500) \\
\hline Secondary & Donkey anti-rabbit & Invitrogen & A31573 & Donkey anti-rabbit (1:500) \\
\hline Vector & Tbr2 Cre & Tyler et al. (2015) & IUE & \\
\hline Vector & Prc1 Cre & Available upon request & IUE & \\
\hline Vector & MFAP2 Cre & Available upon request & IUE & \\
\hline Vector & CAG-StopLight & Montana Molecular & IUE & \\
\hline Vector & CAG-LNL-LYN-GFP & Available upon request & IUE & \\
\hline Software, algorithm & $\mathrm{R}$ & https://www.r-project.org & SCR 001905 & \\
\hline Software, algorithm & Seurat & https:/^illı.org/seurat/ & RRID:SCR_007322 & \\
\hline Software, algorithm & Fiji & https://imagej.net/Fiji & RRID:SCR_002285 & \\
\hline Software, algorithm & Prism & https://www.graphpad.com/scientific-software/prism/ & RRID:SCR_002798 & \\
\hline Software, algorithm & Sigma Plot & https://systatsoftware.com/downloads/download-sigmaplot/ & & \\
\hline Primers & Prc1 forward & TACCGTTCTCCGTCCCGCTCGAGGGGCAGAGCCG & & \\
\hline Primers & Prc1 reverse & GCTCTGCCCCTCGAGCGGGACGGAGAACGG & & \\
\hline Primers & Prc1 forward & GGCGAATTGGGTACCCTTGGCTTGCTAGGGTGTGA & & \\
\hline Primers & Prc1 reverse & CCCTAGCAAGCCAAGCGCTATC & & \\
\hline Primers & Robo4 forward & GGCGAATTGGGTACCCATGCATTTGGAGTTTCCATGTCCT & & \\
\hline Primers & Robo4 reverse & GCTCTGCCCCTCGAGGGCTGCTCTCGGCTCC & & \\
\hline Primers & MFAP2 forward & GGCGAATTGGGTACCACTCGATCTCCCTTAATCTGCCT & & \\
\hline Primers & MFAP2 reverse & GGCGAATTGGGTACCACTCGATCTCCCTTAATCTGCCT & & \\
\hline Primers & ZIKV forward & AARTACACATACCARAACAAAGTGGT & & \\
\hline Primers & ZIKV reverse & TCCRCTCCCYCTYTGGTCTTG & & \\
\hline
\end{tabular}

\section{AXL as a group 1 identifier and candidate viral entry receptor}

Despite evidence that $A X L$ is sufficient but not necessary to enable ZIKV entry (Wells et al., 2016), we sought to test whether aRGC1 vulnerability to ZIKV was correlated with $A X L$ expression. RNAscope of Prc1 $+A X L$ and MFAP2 + AXL corroborated the scRNAseq data and showed that $A X L$ mRNA levels are higher in aRGC1/ $\mathrm{PrC}^{+}{ }^{+}$cells compared with aRGC2/MFAP2 ${ }^{+}$cells (Fig. $8 C, D)$. However, $26 \%$ of $M F A P 2^{+}$cells did express $A X L$, showing that $A X L$ expression is not exclusive to aRGC1 (Fig. 8E,F). When multiplexing $A X L$ with ZIKV and the respective group marker, we found that $A X L, \operatorname{Prc1}$, and ZIKV were co-expressed at high levels (Fig. 8G,H). This correlation between $A X L$ expression and ZIKV infection is consistent with previous findings in vitro (Miner and Diamond, 2016; Nowakowski et al., 2016; Meertens et al., 2017). These findings do not explain why no significant difference has been found between AXL-deficient mice and their littermates reported in Wang et al. (2017), which implicate viral entry receptors other than AXL for ZIKV infection. Future studies should leverage scRNAseq data to mark multiple viral entry receptors with RNAscope to further understand how $A X L$ interacts with other entry receptors in aRGC ZIKV infection.

\section{Discussion}

Determining the mechanisms by which ZIKV alters fetal brain growth and function can enable more focused therapies to support the lives of children with congenital Zika syndrome. Details about the route of infection, the cell types most impacted by the virus, and the temporal change in proliferative and postmitotic zones of the growing brain are needed to provide the best framework for future supportive care for those exposed to ZIKV during fetal life. We therefore developed an in vivo model of ZIKV brain infection, using direct forebrain intraventricular injection, that enables this type of longitudinal, cell typespecific analysis without the need for immune system attenuation or virus inactivation. This approach of infecting the mouse brain at different timepoints in pregnancy and then collecting infected and control embryos 1-6 DPI allowed for a measurement of the progression of ZIKV infection. 
Table 3: Statistics table

\begin{tabular}{|c|c|c|c|c|c|c|c|c|}
\hline Figure & Experiment & $n$ & Data structure & Type of test & $p$ value & SE control & SE infected & SE \\
\hline $3 B$ & $2 \mathrm{DPI} \mathrm{ML}$ & 4 & Normal distribution & $t$ test & 0.00136 & 0.00 & 0.00 & \\
\hline $3 B$ & $3 \mathrm{DPI} M L$ & 4 & Normal distribution & $t$ test & 0.00017204 & 0.00 & 0.01 & \\
\hline $3 C$ & 3 DPI RC & 4 & Normal distribution & $t$ test & 0.0688023 & 0.01 & 0.01 & \\
\hline $3 B$ & $4 \mathrm{DPI} \mathrm{ML}$ & 4 & Normal distribution & $t$ test & 0.0146552 & 0.01 & 0.02 & \\
\hline $3 C$ & $5 \mathrm{DPI} \mathrm{RC}$ & 4 & Normal distribution & $t$ test & 0.03849331 & 0.59 & 0.49 & \\
\hline $3 B$ & 6DPI ML & 4 & Normal distribution & $t$ test & 0.000118 & 0.01 & 0.01 & \\
\hline $3 C$ & $6 \mathrm{DPI} \mathrm{RC}$ & 4 & Normal distribution & $t$ test & 0.00028544 & 0.02 & 0.01 & \\
\hline $3 D$ & $1 \mathrm{DPI} 1 \mathrm{ML}$ & 4 & Normal distribution & $t$ test & 0.00684739 & 0.00 & 0.00 & \\
\hline $3 E$ & 1 DPI $1 \mathrm{RC}$ & 4 & Normal distribution & $t$ test & 0.000128 & 0.00 & 0.00 & \\
\hline $3 D$ & $1 \mathrm{DPI} 2 \mathrm{ML}$ & 4 & Normal distribution & $t$ test & 0.0013651 & 0.01 & 0.01 & \\
\hline $3 E$ & 1 DPI 4 RC & 4 & Normal distribution & $t$ test & 0.00169226 & 0.01 & 0.00 & \\
\hline $3 F$ & $2 \mathrm{DPI} 1 \mathrm{ML}$ & 4 & Normal distribution & $t$ test & 0.00136 & 0.01 & 0.01 & \\
\hline $3 G$ & $2 \mathrm{DPI} 1 \mathrm{RC}$ & 4 & Normal distribution & $t$ test & 0.00349 & 0.00 & 0.01 & \\
\hline $3 F$ & $2 \mathrm{DPI} 2 \mathrm{ML}$ & 4 & Normal distribution & $t$ test & 0.00495933 & 0.01 & 0.01 & \\
\hline $3 G$ & $2 \mathrm{DPI} 2 \mathrm{RC}$ & 4 & Normal distribution & $t$ test & 0.001435 & 0.01 & 0.00 & \\
\hline $3 F$ & $2 \mathrm{DPI} 3 \mathrm{ML}$ & 4 & Normal distribution & $t$ test & 0.00695174 & 0.01 & 0.01 & \\
\hline $3 G$ & 2 DPI 3 RC & 4 & Normal distribution & $t$ test & 0.00914545 & 0.01 & 0.01 & \\
\hline $3 J$ & $1 \mathrm{DPI}$ & 4 & Normal distribution & $t$ test & 0.00180085 & 9.49 & 3.19 & \\
\hline $3 J$ & $2 \mathrm{DPI}$ & 4 & Normal distribution & $t$ test & $2.7878 \mathrm{E}-07$ & 0.64 & 3.83 & \\
\hline $3 J$ & $3 \mathrm{DPI}$ & 4 & Normal distribution & $t$ test & 3.3158E-07 & 3.11 & 2.67 & \\
\hline $3 \mathrm{~J}$ & $4 \mathrm{DPI}$ & 4 & Normal distribution & $t$ test & 3.4267E-06 & 5.12 & 3.79 & \\
\hline $3 \mathrm{~J}$ & $5 \mathrm{DPI}$ & 4 & Normal distribution & $t$ test & 3.3551E-05 & 6.57 & 5.81 & \\
\hline $3 \mathrm{~J}$ & $6 \mathrm{DPI}$ & 4 & Normal distribution & $t$ test & 3.865E-06 & 6.14 & 2.29 & \\
\hline $4 G$ & Bin 2 & 4 & Normal distribution & $t$ test & 0.02065501 & 1.04 & 1.19 & \\
\hline $4 G$ & Bin 3 & 4 & Normal distribution & $t$ test & 0.07577485 & 1.29 & 0.85 & \\
\hline $4 G$ & Bin 4 & 4 & Normal distribution & $t$ test & 0.1339746 & 1.11 & 0.00 & \\
\hline $5 G$ & Non-Tbr2 & 4 & Normal distribution & $t$ test & $2.454 \mathrm{E}-06$ & 0.68 & 1.17 & \\
\hline $5 H$ & Ph3 Tbr2 & 4 & Normal distribution & $t$ test & 0.01009862 & 0.01 & 0.00 & \\
\hline $5 /$ & Ph3 non Tbr2 & 4 & Normal distribution & $t$ test & 2.6496E-06 & 0.01 & 0.01 & \\
\hline $5 J$ & Ph3 Tbr2 & 4 & Normal distribution & $t$ test & 0.00071178 & 0.15 & 1.04 & \\
\hline $5 K$ & Ph3 sox2 & 4 & Normal distribution & $t$ test & 0.00016526 & 0.83 & 0.38 & \\
\hline $5 L$ & $1 \mathrm{DPI}$ & 4 & Normal distribution & $t$ test & 7.7206E-06 & 1.26 & 0.38 & \\
\hline $5 L$ & $2 \mathrm{DPI}$ & 4 & Normal distribution & $t$ test & $1.4818 \mathrm{E}-07$ & 3.21 & 1.46 & \\
\hline $5 L$ & $3 \mathrm{DPI}$ & 4 & Normal distribution & $t$ test & 0.00011085 & 2.25 & 1.70 & \\
\hline $5 L$ & $4 \mathrm{DPI}$ & 4 & Normal distribution & $t$ test & 0.59430986 & 1.18 & 1.59 & \\
\hline $5 L$ & $5 \mathrm{DPI}$ & 4 & Normal distribution & $t$ test & 0.90448279 & 1.43 & 1.05 & \\
\hline $5 L$ & $6 \mathrm{DPI}$ & 4 & Normal distribution & $t$ test & 0.27823572 & 0.31 & 0.38 & \\
\hline $5 M$ & $1 \mathrm{DPI}$ & 4 & Normal distribution & $t$ test & 6.4046E-06 & 0.78 & 0.25 & \\
\hline $5 M$ & $2 \mathrm{DPI}$ & 4 & Normal distribution & $t$ test & 0.00029411 & 1.78 & 1.29 & \\
\hline $5 M$ & $3 \mathrm{DPI}$ & 4 & Normal distribution & $t$ test & 0.00011655 & 1.25 & 2.24 & \\
\hline $5 M$ & $4 \mathrm{DPI}$ & 4 & Normal distribution & $t$ test & 0.32147813 & 1.66 & 1.10 & \\
\hline $5 M$ & $5 \mathrm{DPI}$ & 4 & Normal distribution & $t$ test & 0.24536482 & 1.10 & 1.21 & \\
\hline $5 M$ & $6 \mathrm{DPI}$ & 4 & Normal distribution & $t$ test & 0.53696332 & 0.12 & 0.06 & \\
\hline $6 B$ & endfeet & 4 & Normal distribution & $t$ test & 0.03248771 & 0.03 & 0.02 & \\
\hline $6 G$ & Dil & 4 & Normal distribution & $\begin{array}{l}t \text { test } \\
\text { htinued) }\end{array}$ & 0.00082241 & 0.04 & 0.05 & \\
\hline
\end{tabular}


Table 3: Continued

\begin{tabular}{|c|c|c|c|c|c|c|c|c|}
\hline Figure & Experiment & $n$ & Data structure & Type of test & $p$ value & SE control & SE infected & SE \\
\hline$\overline{7 D}$ & Prc1 24 h & 4 & Normal distribution & $t$ test & $1.5942 \mathrm{E}-10$ & 1.29 & 1.64 & \\
\hline $7 I$ & MFAP2 $24 \mathrm{~h}$ & 4 & Normal distribution & $t$ test & 8.9982E-11 & 0.69 & 0.95 & \\
\hline 70 & IUE and RNAscope & 4 & Normal distribution & $t$ test & 8.0791E-11 & 0.10 & 0.98 & \\
\hline $7 Q$ & Double RNAscope & 4 & Normal distribution & ANOVA & 0.0001 & 1.08 & 1.81 & 1.05 \\
\hline $8 B$ & MFAP2 Prc1 infected & 4 & Normal distribution & $t$ test & 1.3469E-05 & 1.37 & 0.88 & \\
\hline $8 H$ & Prc1 AXL ZIKV & 4 & Normal distribution & $t$ test & $4.8802 \mathrm{E}-12$ & 0.92 & 0.27 & \\
\hline
\end{tabular}

The concentration and strain of virus used here uncovered less cell death and more morphologic differences in the developing cells in the forebrain. We found that ZIKV passes first through a subpopulation of aRGCs expressing high levels of $A X L$, then transits to intermediate progenitors. Furthermore, while the viral infection causes devastating loss to certain cell groups, other precursors and postmitotic neurons appear less affected. Even at the latest time points examined, key structural features of the neocortex are maintained, and many neurons survive despite harboring viral infection.

The ability to assess infection across time enabled us to determine whether the severity of outcome depends on the age at which infection first occurs. Surprisingly, the severity of microencephaly was not dependent on the developmental stage during infection. The consistent microencephaly observed across developmental stages may be a consequence of the under-developed mouse immune system, as the first immune response to antigens is not found until the first postnatal week (Holsapple et al., 2003). Measurements of cell death and proliferation showed that viral infection increases the number of cells marked for apoptosis while decreasing the number of proliferating cells, both of which can result in microencephaly. The disruption in aRGC basal fibers likely contributes to the microencephaly phenotype as well by interrupting this key substrate of neuronal migration. This interference in migration may also play a role in the cell death seen postnatally (Dudley et al., 2017).

Our model allowed for in vivo time course studies to measure the progression of ZIKV infection over the course of $6 \mathrm{~d}$ of prenatal brain development. This is the longest in vivo time course study of a ZIKV model to date. This allowed for tracking of the microencephaly phenotype over time and elucidated some surprising results. We found that severity of microencephaly in this model was consistent across DPI1-DPI6. One explanation for this is that certain aRGCs are targeted initially, creating a severe depletion in their proliferation and neurogenesis which is then compensated for by the remaining aRGCs and other NPC types. Alternatively, as soon as new aRGCs are born and able to proliferate, cortical expansion continues at the same rate, but is overall unable to compensate for the initial volume loss.

It was also surprising that the microencephaly phenotype was not impacted by the age of the animal at the start of infection given that human data shows increased severity of infections occurring earlier in pregnancy (Johansson et al., 2016; Schuler-Faccini et al., 2016). This is likely because of the differences in human and murine immune development, to species-specific differences in plasticity of the germinal zones, or to the condensed timeframe of cortical development in mouse compared with human. Unlike humans, mice do not have fully developed immune systems until one week after birth. Thus, there may be additional inflammatory effects on human brain following ZIKV infection compared with mouse. The E13.5-E16.5 time frame covers the peak of mouse neurogenesis. Some differences would have been expected between E13.5 and E16.5, however, it may be possible that the difference in aRGC number is not great enough in this narrow window to see differences. If it were more technically possible, starting the IUE timepoints even earlier would have helped further explain the progression of ZIKV infection in causing microencephaly. Additionally, a proliferation compensation mechanism may be in play. This is an interesting avenue for future study given the role that cell-to-cell contact plays in proliferation. If cell density in the germinal zones is decreased because of ZIKV-induced cell death, the decreased pressure on cell contact could allow for transiently increased proliferation in the unaffected cell populations. While ours and other data suggest decreased proliferation overall, longer time courses of proliferation still need to be tested.

The cell type vulnerability identified in this study identifies precursor groups that differ in their susceptibility to the virus. For example, we found greater defects in proliferation and morphologic development in apical precursor cells, which are likely responsible for the rapid expression of microencephaly within a day after infection. While aRGCs are infected preferentially at 1-2 DPI, bIPCs were the largest infected population at 3 DPI. This may explain the discrepancy in previous findings of aRGCs being the primary target versus others who found IPCs to be ZIKV's primary target (Onorati et al., 2016; Lin et al., 2017). However, as microencephaly did not significantly worsen over time after infection in the mouse, the initial and substantial impact on proliferation appears to be compensated by continued proliferation in the remaining aRGCs and IPCs. Fate mapping with the IUE-based Cre/LoxP recombination system identified that diversity in the aRGC population may underlie growth dynamics in brains infected with ZIKV. In particular, we provide evidence that $\mathrm{MFAP}^{+}$aRGCs are less susceptible to ZIKV infection, indicating that this group of cells, and their progeny, may 
underlie the compensatory proliferation described above. One possible mechanism responsible for the differential vulnerability between $P r c 1^{+}$cells and MFAP2 ${ }^{+}$cells is the increased expression of $A X L$ in the Prc1+ cells. However, these findings also suggest that $A X L$ is not necessary for infection, as $4 \%$ of $Z \mathrm{ZIVV}^{+}$cells were $A X L^{-}$. The presented data corroborate the findings of Nowakowski et al. (2016) who showed that $A X L$ is highly expressed in aRGCs and functions as a viral entry receptor; these data are also supportive of the results of Wells et al. (2016), which demonstrated that cells could become infected without the presence of $A X L$ (Onorati et al., 2016; Wells et al., 2016). One $A X L$-independent means of viral infection could be the passage of viral RNA during cell division. Although these results as well as previous research indicate that mitosis is impaired during infection (Onorati et al., 2016; Tang et al., 2016; Dudley et al., 2017; Lin et al., 2017; Rosenfeld et al., 2017), if an already infected aRGC is able to successfully divide, it could pass infection to its bIPC daughter cell. Nevertheless, the high correlation between Prc1 expression, $A X L$ expression, and ZIKV infection make $A X L$ an interesting group 1 candidate marker gene. This model provides a framework for additional candidate viral entry research aimed at designing chemical inhibitors. It also suggests that interference of AXL-mediated endocytosis will decrease infection rates in the highly targeted aRGC1 population, potentially avoiding the severe and early onset of microcephaly as indicated by previous work showing decreased ZIKV infection from decreased AXL expression (Wells et al., 2016).

These results not only increase understanding of how ZIKV infection impacts neural development, but also indicate that a subtype of aRGCs, which previously were believed to be a largely homogenous population, may be critically involved in the initiation of infection. Li et al. (2020) showed transcriptional priming and pseudotime evidence that aRGC1 $\left(\mathrm{PrC}^{+}\right)$may be fated toward direct neurogenesis while aRGC2 $\left(M F A P 2^{+}\right)$cells are primed toward the $\mathrm{Tbr2}^{+}$bIPC lineage (Li et al., 2020). Similarly, another study recently described aRGC heterogeneity based on Sox9 expression (Fabra-Beser et al., 2020), which is also differentially expressed between aRGC1 and aRGC2 groups (log fold change: -0.62 , adjusted $p$ value: 0.00022 ). Future work using tools designed to study cortical circuits subserved by aRGC1 and aRGC2 progeny will highlight specific changes in neural architecture likely disturbed in people with congenital Zika syndrome.

\section{References}

Bhattacharyya S, Zagórska A, Lew ED, Shrestha B, Rothlin CV, Naughton J, Diamond MS, Lemke G, Young JA (2013) Enveloped viruses disable innate immune responses in dendritic cells by direct activation of TAM receptors. Cell Host Microbe 14:136-147.

Center for Disease Control (2017) CDC study estimates 20-fold increase in certain types of birth defects in pregnancies with possible Zika infection compared with pre-Zika years. Available at https:// www.cdc.gov/media/releases/2017/s0302-zika-birth-defects.html.

Dudley DM, Newman CM, Lalli J, Stewart LM, Koenig MR, Weiler AM, Semler MR, Barry GL, ZarbockKR, Mohns MS, Breitbach ME, Schultz-Darken N, Peterson E, Newton W, Mohr EL, Capuano lii S, Osorio JE, O'Connor SL, O'ConnorDH, Friedrich TC, et al. (2017)
Infection via mosquito bite alters Zika virus tissue tropism and replication kinetics in rhesus macaques. Nat Commun 8:2096.

Englund C, Fink A, Lau C, Pham D, Daza RA, Bulfone A, Kowalczyk T, Hevner RF (2005) Pax6, Tbr2, and Tbr1 are expressed sequentially by radial glia, intermediate progenitor cells, and postmitotic neurons in developing neocortex. J Neurosci 25:247-251.

Fabra-Beser J, Alves Medeiros de Araujo J, Marques-Coelho D, Goff LA, Müller U, Gil-Sanz C (2020) Differential expression levels of Sox9 in early neocortical radial glial cells regulate the decision between stem cell maintenance and differentiation. bioRxiv 417931.

Gadea G, Bos S, Krejbich-Trotot P, Clain E, Viranaicken W, ElKalamouni C, Mavingui $P$, Desprès $P$ (2016) A robust method for the rapid generation of recombinant Zika virus expressing the GFP reporter gene. Virology 497:157-162.

Hartfuss E, Galli R, Heins N, Götz M (2001) Characterization of CNS precursor subtypes and radial glia. Dev Biol 229:15-30.

Haubensak W, Attardo A, Denk W, Huttner WB (2004) Neurons arise in the basal neuroepithelium of the early mammalian telencephaIon: a major site of neurogenesis. Proc Natl Acad Sci USA 101:3196-3201.

Heavner WE, Ji S, Notwell JH, Dyer ES, Tseng AM, Birgmeier J, Yoo B, Bejerano G, McConnell SK (2020) Transcription factor expression defines subclasses of developing projection neurons highly similar to single-cell RNA-seq subtypes. Proc Natl Acad Sci USA 117:25074-25084.

Hodge RD, Bakken TE, Miller JA, Smith KA, Barkan ER, Graybuck LT, Close JL, Long B, Johansen N, Penn O, Yao Z, Eggermont J, Höllt T, Levi BP, Shehata SI, Aevermann B, Beller A, Bertagnolli D, Brouner K, Casper T, et al. (2019) Conserved cell types with divergent features in human versus mouse cortex. Nature 573:61-68.

Holsapple MP, West LJ, Landreth KS (2003) Species comparison of anatomical and functional immune system development. Birth Defects Res B Dev Reprod Toxicol 68:321-334.

Johansson MA, Mier-y-Teran-Romero L, Reefhuis J, Gilboa SM, Hills SL (2016) Zika and the risk of microcephaly. N Engl J Med 375:14.

Lazear HM, Govero J, Smith AM, Platt DJ, Fernandez E, Miner JJ, Diamond MS (2016) A mouse model of Zika virus pathogenesis. Cell Host Microbe 19:720-730.

Li C, Lin M, Liu J (2004) Identification of PRC1 as the p53 target gene uncovers a novel function of p53 in the regulation of cytokinesis. Oncogene 23:9336-9347.

Li Z, Tyler WA, Zeldich E, Santpere Baró G, Okamoto M, Gao T, Li M, Sestan N, Haydar TF (2020) Transcriptional priming as a conserved mechanism of lineage diversification in the developing mouse and human neocortex. Sci Adv 6:eabd2068.

Lin MY, Wang YL, Wu WL, Wolseley V, Tsai MT, Radic V, Thornton ME, Grubbs BH, Chow RH, Huang IC (2017) Zika virus infects intermediate progenitor cells and post-mitotic committed neurons in human fetal brain tissues. Sci Rep 7:14883.

Meertens L, Labeau A, Dejarnac O, Cipriani S, Sinigaglia L, BonnetMadin L, Le Charpentier T, Hafirassou ML, Zamborlini A, CaoLormeau VM, Coulpier M, Missé D, Jouvenet N, Tabibiazar R, Gressens P, Schwartz O, Amara A (2017) AxI mediates ZIKA virus entry in human glial cells and modulates innate immune responses. Cell Rep 18:324-333.

Miner JJ, Diamond MS (2016) Understanding how Zika virus enters and infects neural target cells. Cell Stem Cell 18:559-560.

Miyata T, Kawaguchi A, Saito K, Kawano M, Muto T, Ogawa M (2004) Asymmetric production of surface-dividing and non-surface-dividing cortical progenitor cells. Development 131:31333145.

Noctor SC, Martínez-Cerdeño V, Ivic L, Kriegstein AR (2004) Cortical neurons arise in symmetric and asymmetric division zones and migrate through specific phases. Nat Neurosci 7:136-144.

Nowakowski TJ, Pollen AA, Di Lullo E, Sandoval-Espinosa C, Bershteyn M, Kriegstein AR (2016) Expression analysis highlights $\mathrm{AXL}$ as a candidate Zika virus entry receptor in neural stem cells. Cell Stem Cell 18:591-596. 
Okada Y, Naruse H, Tanaka T, Funahashi N, Regan ER, Yamakawa K, Hino N, Ishimoto K, Doi T, Aird WC (2015) Expression of the Robo4 receptor in endothelial cells is regulated by two AP-1 protein complexes. Biochem Biophys Res Commun 467:987-991.

Onorati M, Li Z, Liu F, Sousa AMM, Nakagawa N, Li M, Dell'Anno MT, Gulden FO, Pochareddy S, Tebbenkamp ATN, Han W, Pletikos M, Gao T, Zhu Y, Bichsel C, Varela L, Szigeti-Buck K, Lisgo S, Zhang Y, Testen A, Gao XB, et al. (2016) Zika virus disrupts phospho-TBK1 localization and mitosis in human neuroepithelial stem cells and radial glia. Cell Rep 16:2576-2592.

Politi LS, Salsano E, Grimaldi M (2020) Magnetic resonance imaging alteration of the brain in a patient with coronavirus disease 2019 (COVID-19) and anosmia. JAMA Neurol 77:1028.

Rakic P (1972) Mode of cell migration to the superficial layers of fetal monkey neocortex. J Comp Neurol 145:61-83.

Rakic P (1987) Neurobiology of neocortex: report of the Dahlem workshop on neurobiology of neocortex (Rakic P and Singer W, eds), pp 5-27. Berlin: Wiley Limited.

Rakic P (1988) Specification of cerebral cortical areas. Science 241:170-176.

Rakic P (1995) A small step for the cell, a giant leap for mankind: a hypothesis of neocortical expansion during evolution. Trends Neurosci 18:383-388.

Rosenfeld AB, Doobin DJ, Warren AL, Racaniello VR, Vallee RB (2017) Replication of early and recent Zika virus isolates throughout mouse brain development. Proc Natl Acad Sci USA 114:12273-12278.

Rossignol ED, Peters KN, Connor JH, Bullitt E (2017) Zika virus induced cellular remodelling. Cell Microbiol 19:10.1111/cmi.12740.

Schuler-Faccini L, Sanseverino M, Vianna $F$, da Silva AA, Larrandaburu M, Marcolongo-Pereira C, Abeche AM (2016) Zika virus: A new human teratogen? Implications for women of reproductive age. Clin Pharmacol Ther 100:28-30.
Shao Q, Herrlinger S, Yang S, Lai F, Moore JM, Brindley M, Chen J (2016) Zika virus infection disrupts neurovascular development and results in postnatal microcephaly with brain damage. Development 143:4127-4136.

Tamamaki N, Nakamura K, Okamoto K, Kaneko T (2001) Radial glia is a progenitor of neocortical neurons in the developing cerebral cortex. Neurosci Res 41:51-60.

Tang H, Hammack C, Ogden SC, Wen Z, Qian X, Li Y, Yao B, Shin J, Zhang F, Lee EM, Christian KM, Didier RA, Jin P, Song H, Ming GL (2016) Zika virus infects human cortical neural progenitors and attenuates their growth. Cell Stem Cell 18:587-590.

Tasic B, Yao Z, Graybuck LT, Smith KA, Nguyen TN, Bertagnolli D, Goldy J, Garren E, Economo MN, Viswanathan S, Penn O, Bakken $\mathrm{T}$, Menon V, Miller J, Fong O, Hirokawa KE, Lathia K, Rimorin C, Tieu M, Larsen R, et al. (2018) Shared and distinct transcriptomic cell types across neocortical areas. Nature 563:72-78.

Tyler WA, Haydar TF (2013) Multiplex genetic fate mapping reveals a novel route of neocortical neurogenesis, which is altered in the Ts65Dn mouse model of Down syndrome. J Neurosci 33:51065119.

Tyler WA, Medalla M, Guillamon-Vivancos T, Luebke JI, Haydar TF (2015) Neural precursor lineages specify distinct neocortical pyramidal neuron types. J Neurosci 35:6142-6152.

Vanwalscappel B, Tada T, Landau NR (2018) Toll-like receptor agonist R848 blocks Zika virus replication by inducing the antiviral protein viperin. Virology 522:199-208.

Wang ZY, Wang Z, Zhen ZD, Feng KH, Guo J, Gao N, Fan DY, Han DS, Wang PG, An J (2017) Axl is not an indispensable factor for Zika virus infection in mice. J Gen Virol 98:2061-2068.

Wells MF, Salick MR, Wiskow O, Ho DJ, Worringer KA, Ihry RJ, Kommineni S, Bilican B, Klim JR, Hill EJ, Kane LT, Ye C, Kaykas A, Eggan K (2016) Genetic ablation of AXL does not protect human neural progenitor cells and cerebral organoids from Zika virus infection. Cell Stem Cell 19:703-708. 